\title{
Stark profiles of infrared helium lines
}

\author{
N. Terzi ${ }^{1}$, S. Sahal-Bréchot ${ }^{2}$, and Z. Ben Lakhdar ${ }^{1}$ \\ 1 Laboratoire de Physique Atomique et Moléculaire, Département de Physique, Faculté des Sciences de Tunis, 1060 Campus \\ Universitaire, Tunis, Tunisie \\ 2 Laboratoire "Atomes et Molécules en Astrophysique", Unité associée au CNRS 812, Département Atomes et Molécules en \\ Astrophysique, Observatoire de Paris-Meudon, 92195 Meudon Cedex, France
}

Received April 20; accepted May 29, 1998

\begin{abstract}
We have calculated Stark widths and shifts of some infrared helium lines that are missing and that are important for modellisation of the atmospheres of hot early-type stars. These calculations are based on the impact and quasistatic theory and semi-classical approximation. The provided data are displayed for a grid of temperatures $\left(10^{4}\right.$ to $\left.10^{5} \mathrm{~K}\right)$ and electron densities $\left(10^{10}\right.$ to $\left.10^{13} \mathrm{~cm}^{-3}\right)$ typical of hot early-type stars. We also discuss the conditions of use of our data for astrophysical purposes.
\end{abstract}

Key words: stars: early-type — infrared: general — atomic data - lines: profiles

\section{Introduction}

Stark broadening of spectral lines is important for the modellisation of the atmospheres of hot early-type stars, where these lines are strong and can have extended collisional wings due to interactions with electrons, protons and also ionised helium. In spite of numerous efforts oriented towards the obtention of atomic parameters entering the calculation of spectral lines intensities and profiles, a number of such important parameters are still missing. For helium lines, the atomic parameters which are available are numerous (cf. for instance Dimitrijević \& SahalBréchot 1984), but they do not concern lines arising from highly excited levels. In particular, there are six infrared helium lines which perturb the wings of Paschen hydrogen lines observed in hot early-type stars. These hydrogen lines are an efficient tool for determining stellar atmospheres parameters (Jaschek et al. 1994; Frémat et al. 1996). Thus the line profiles of the perturbing helium lines must be known with a sufficient precision for an accurate modellisation of these stellar atmospheres. In the present

$\overline{\text { Send offprint requests to }}$ : S. Sahal-Bréchot (sahal@obspm.fr) paper, we present results for Stark widths and shifts for these six infrared lines of helium that have never been calculated before. We have used the impact and quasistatic approximations, and the semi-classical perturbation theory that we have shown to be sufficient for these lines connecting a high and a low level. We have also shown that the isolated line approximation can be used in spite of the high levels involved.

\section{Theory for isolated lines}

Using the Sahal-Bréchot code based on the Sahal-Bréchot theory (Sahal-Bréchot 1969, cf. also Baranger 1958a-c for the basic theory) and constanly updated (cf. for example Ben Nessib et al. 1996), which assumes: the impact approximation, the semi-classical approximation (the radiator is treated quantically and the perturbers follow classical paths, which are straight lines in the case of neutral radiating atoms) and the perturbation theory, we have calculated the full half width $2 W$ and the shift $d$ of the line emitted between the initial level $i$ and the final level $f$ which are given by the following formulae (Sahal-Bréchot 1969):

$$
\begin{aligned}
& 2 W=N \int_{0}^{\infty} v f(v) \mathrm{d} v\left(\sum_{i^{\prime} \neq i} \sigma_{i i^{\prime}}(v)+\sum_{f^{\prime} \neq f} \sigma_{f f^{\prime}}(v)+\sigma_{\mathrm{el}}\right)(1) \\
& d=\int_{0}^{\infty} v f(v) \mathrm{d} v \int_{R_{3}}^{R_{\mathrm{d}}} 2 \pi \rho \mathrm{d} \rho \sin 2 \phi_{\mathrm{p}}
\end{aligned}
$$

$i^{\prime}$ and $f^{\prime}$ are the perturbing levels, $N$ and $v$ are respectively the density and the velocity of perturbers, $f(v)$ is the Maxwellian distribution of velocities.

The inelastic cross sections $\sigma_{i i^{\prime}}(v)$ (respectively $\left.\sigma_{f f^{\prime}}(v)\right)$ can be expressed by an integration over the impact parameter $\rho$ of the transition probability $P_{i i^{\prime}}$ as

$\sum_{i^{\prime} \neq i} \sigma_{i i^{\prime}}(v)=\frac{1}{2} \pi R_{1}^{2}+\int_{R_{1}}^{R_{\mathrm{d}}} 2 \pi \rho \mathrm{d} \rho \sum_{i^{\prime} \neq i} P_{i i^{\prime}}(\rho, v)$. 
The elastic collision contribution to the width is given by: $\sigma_{\mathrm{el}}=2 \pi R_{2}^{2}+\int_{R_{2}}^{R_{\mathrm{d}}} 8 \pi \rho \mathrm{d} \rho \sin ^{2} \delta$

$\delta=\left(\phi_{\mathrm{p}}^{2}+\phi_{\mathrm{q}}^{2}\right)^{\frac{1}{2}}$.

The phase shifts $\phi_{\mathrm{p}}$ and $\phi_{\mathrm{q}}$ are due respectively to the polarisation and the quadrupole potential (Sahal-Bréchot 1969).

The cut-offs $R_{1}, R_{2}, R_{3}$, the Debye cut-off $R_{\mathrm{d}}$ and the symmetrization procedures are described by SahalBréchot (1969) and further papers and will not be rediscussed therein.

As already discussed by Baranger (1958a-c, 1962) and Sahal-Bréchot (1969), the impact approximation is valid when the average effect of collisions is weak, in other words when strong collisions are separated in time, or equivalently, when the duration of a collision is very small compared to the separation time between strong collisions. The validity criterion for the impact approximation is given by:

$C_{1}=\tau W_{\text {strong }} \ll 1$

where $\tau$ is a typical collision duration and $W_{\text {strong }}$ is the strong collision contribution to the collisional half-width. It can also be written as (Ben Nessib et al. 1996):

$C_{1}=N \pi \rho_{\text {typ }}^{3} \ll 1$

where $\rho_{\text {typ }}$ is a typical impact parameter for strong collisions.

The resulting profiles are Lorentzian. This condition is well verified by electronic collisions for a large range of densities.

For ionic collisions the impact approximation fails, especially for high densities. Then we can apply the quasistatic approximation. If the quadratic interaction potential is dominant, the quasistatic total width and shift are given by (Griem 1974):

$W_{\mathrm{iq}}=1.7510^{-4} N_{\mathrm{e}}^{\frac{1}{4}} A\left[1-0.068 N_{\mathrm{e}}^{\frac{1}{6}} T^{-\frac{1}{2}}\right] W_{\mathrm{e}}$

$d_{\text {iq }}=10^{-4} N_{\mathrm{e}}^{\frac{1}{4}} A\left[1-0.068 N_{\mathrm{e}}^{\frac{1}{6}} T^{-\frac{1}{2}}\right] W_{\mathrm{e}}$

$W_{\mathrm{e}}$ is the impact contribution of electrons to the total width.

$A$ is the quasistatic quadratic parameter, defined by Griem (1964), Traving (1968) and expressed by Ben Nessib et al. (1996) as follows:

$A=\left(\frac{e F_{0}^{2}}{\hbar W_{\mathrm{e}}}\left|\alpha_{i}-\alpha_{f}\right|\right)^{\frac{3}{4}}$

$F_{0}=2 \pi(4 / 15)^{(2 / 3)} e N_{\mathrm{e}}^{2 / 3}$ is the normal field strength (Griem 1974). $T$ is in Kelvin and $N_{\mathrm{e}}$ in $\mathrm{cm}^{-3}$.

$\alpha_{i}$ is the polarizability of the initial level (resp. $\alpha_{\mathrm{f}}$ is the polarisability of the final level). It is expressed as:

$\alpha_{i}=4 a_{0}^{3} \sum_{i^{\prime} \neq i} f_{i i^{\prime}}\left(\frac{I_{\mathrm{H}}}{\Delta E_{i i^{\prime}}}\right)^{2}$

$a_{0}$ is the Bohr radius and $I_{\mathrm{H}}$ is the ionisation energy of hydrogen.

\section{Application to some infrared neutral He lines}

\subsection{Atomic data}

We give in the following the wavelengths of the studied Helium lines, the corresponding transitions, and the perturbing levels $i^{\prime}$ and $f^{\prime}$.

\begin{tabular}{llll}
\hline$\lambda(\AA)$ & $i-f$ & $i^{\prime}$ levels & $f^{\prime}$ levels \\
\hline 10138 & $7^{1} \mathrm{D}-3^{1} \mathrm{P}$ & $8 f, 7 f, 6 f$, & $5 d, 4 d, 3 d$, \\
& & $8 p, 7 p, 6 p$ & $4 s, 3 s, 2 s$ \\
\hline 10028 & $7^{3} \mathrm{~F}-3^{3} \mathrm{D}$ & $8 g, 7 g, 6 g$, & $5 f, 4 f$, \\
& & $8 d, 7 d, 6 d$ & $5 p, 4 p, 3 p$ \\
\hline 8779 & $9^{3} \mathrm{D}-3^{3} \mathrm{P}$ & $10 f, 9 f, 8 f$, & $5 d, 4 d, 3 d$, \\
& & $10 p, 9 p, 8 p$ & $4 s, 3 s, 2 s$ \\
\hline 8736 & $12^{3} \mathrm{~F}-3^{3} \mathrm{D}$ & $13 g, 12 g, 11 g$, & $5 f, 4 f$, \\
& & $13 d, 12 d, 11 d$ & $5 p, 4 p, 3 p$ \\
\hline 8584 & $10^{3} \mathrm{D}-3^{3} \mathrm{P}$ & $11 f, 10 f, 9 f$, & $5 d, 4 d, 3 d$, \\
& & $11 p, 10 p, 9 p$ & $4 s, 3 s, 2 s$ \\
\hline
\end{tabular}

The energy levels have been taken from Martin (1973). For the $g$-levels which are missing, we have calculated the energies by using an asymptotic expression of the quantum defect and the polarisation potential (Deutsch 1969).

Calculations of oscillator strengths which enter the expressions of the semi-classical cross-sections and the $A$ parameters have been obtained with the Bates \& Damgaard approximation. Owing to the high levels involved, this approximation is sufficient. Moreover these transitions and the corresponding high levels are not included in the recent and sophisticated data of TOPbase (Cunto et al. 1993). Thus for the calculations of the oscillator strengths we have updated a code given by Dimitrijević who used the tables by Oertel \& Shomo (1968) and the Van Regemorter et al. (1979) formula adapted for high levels. For the transitions between the very high levels $n f-n^{\prime} g\left(n, n^{\prime}>7\right)$, we have used the Coulomb approximation, the corresponding quantum defects being negligible.

\subsection{Results and discussion}

\subsubsection{Calculations}

The calculations have been made for a grid of temperatures $\left(10^{4}\right.$ to $\left.10^{5} \mathrm{~K}\right)$ and electronic densities $\left(10^{10}\right.$ to $10^{13} \mathrm{~cm}^{-3}$ ) typical of stellar atmospheres conditions of hot early-type stars. The results are displayed in Tables 1-12 for the six studied helium lines. We give the calculated impact widths $W$ and the shifts $d$ for collisions with electrons, protons, $\mathrm{He}^{+}$and $\mathrm{He}^{++}$ions and the quasistatic parameter $A$. We give also the various validity criterions useful for the following discussion.

$C_{1}$ is the impact validity criterion.

$C_{2}=\frac{W_{\mathrm{inel}}}{W_{\mathrm{el}}}$ gives the ratio of the contribution of inelastic collisions versus elastic ones for the width. 
$C_{3}$ is the criterion for the "one state" approximation validity criterion.

$C_{4}$ is the ratio of the contribution of strong collisions to the total impact width and thus gives a validity criterion for the perturbation theory approximation.

$C_{5}$ is the criterion for the isolated line approximation, defined by (Dimitrijević \& Sahal Bréchot 1984; Baranger 1958b).

$C_{5}=W /\left(\Delta E_{i i^{\prime}}\right)_{\min }$,

where $W$ is the total width,

$W=W_{\mathrm{e}}+0.9 W_{\mathrm{H}^{+}}+0.1 W_{\mathrm{He}^{+}}$,

$W_{\mathrm{e}}$ is the width due to electron collisions, $W_{\mathrm{H}^{+}}$that due proton collisions and $W_{\mathrm{He}^{+}}$that due to collisions with $\mathrm{He}^{+}$. We neglect collisions with $\mathrm{He}^{++}$in this criterion and we assume that protons and $\mathrm{He}^{+}$ions are in proportion of $90 \%$ and $10 \%$ respectively.

$\left(\Delta E_{i i^{\prime}}\right)_{\min }$ is the energy distance between the upper level and the nearest perturbing level.

\subsubsection{Validity criterions of impact and quasistatic approximations}

Impact approximation is always valid for electron collisions, because the corresponding values of $C_{1}$ are very small compared to unity for all temperatures and densities of interest. In fact it can break down for collisions with ions at low temperatures and high densities (we consider that for $C_{1}>0.5$ the impact approximation fails in the line center). It breaks down especially in the wings where the quasistatic approximation condition becomes $\tau \Delta \omega \gg 1$ where $\Delta \omega$ is the detuning. In astrophysics the detuning of interest is very much larger than the collisional half-width because the wings are sensitive to collisions whereas the line center is dominated by the Doppler broadening. Consequently the collisional wings, due to interactions with ions must often be treated within the quasistatic approximation. For obtaining this quasistatic contribution, we have to determine the predominant interaction of interest. For that we argue by looking at the impact regime and we extrapolate to the quasistatic one: if inelastic collisions are dominant $\left(C_{2} \gg 1\right.$ of Tables 112) for the broadening of the studied lines in the impact approximation, we will consider that the same dipolar interaction can be used in the quasistatic regime: then the so-called linear Stark effect is to be used. On the contrary, if elastic collisions are dominant $\left(C_{2} \ll 1\right)$ for the broadening of the studied lines, we look at the relative importance of the quadrupolar and polarisation ("quadratic interaction") potential. We have verified that the quadrupolar interaction is always negligible for the all lines studied in this paper. This is due to the fact that the upper levels of the lines studied are highly excited. Thus the broadening by elastic collisions is entirely due to the polarization potential. This corresponds to the quadratic Stark effect in the quasistatic regime. In that case the $A$ parameter
Griem (1974) is relevant for obtaining the contribution of the ions to the total width.

Our calculations show that for all studied lines, when the impact approximation is not valid $\left(C_{1}>1\right)$, the elastic collisions are generally dominant $\left(C_{2}<1\right)$. Consequently in most cases, the quasistatic effect is quadratic. The opposite case $\left(C_{2}>1\right)$ only occurs for high densities and for low temperatures, for the two following lines: $8651 \AA$ for collisions with $\mathrm{He}^{++}$and $10028 \AA$ for collisions with protons, $\mathrm{He}^{+}$and $\mathrm{He}^{++}$. For these two cases of minor importance, the quasistatic treatment involves the dipolar interaction and is not treated in the present paper.

\subsubsection{Isolated lines and overlapping lines cases}

According to Dimitrijević \& Sahal-Bréchot (1984), if $C_{5}<$ 1 , energy levels broadened by collisions do not overlap. Then the line is isolated. If $C_{5}>1$ we have in principle to treat the problem of overlapping lines. We show that, for all the studied lines of the present paper, the problem does not appear. The isolated line criterion is always satisfied for the lines: $10138 \AA$, $8779 \AA$, $8651 \AA$, and $8584 \AA$. In fact, the criterion is not satisfied for the line $10028 \AA$ at densities equal or higher than $10^{12} \mathrm{~cm}^{-3}$. For the $8736 \AA$ line, the criterion is not satisfied for densities equal or higher than $10^{11} \mathrm{~cm}^{-3}$. However, if we look at the ratio of the contribution of the upper level to that of lower level to the impact width (the $C_{3}$ criterion), we conclude that the "one state" approximation is always valid, because the lower level contribution is always negligible $\left(C_{3} \gg 1\right)$. Therefore the off-diagonal elements (Baranger 1958b) which enter the expression of the line profiles of overlapping lines in the "two state case" can be neglected. Consequently it is possible to continue to use the isolated line approximation in the "one state case" for the present studied lines which should be a priori not isolated.

In addition, we have checked the validity condition of the perturbation theory. The validity criterion is given by $C_{4}$, which represents the relative contribution of strong collisions to the total impact width. It is well satisfied $\left(C_{4}\right.$ does not exceed a few 20\%) for collisions with electrons. For collisions with ions, the strong collision contribution is more important $\left(C_{4}\right.$ is of the order of $\left.65 \%\right)$ and the perturbation approximation is not so good.

Finally, it can be noticed that the ionic shifts of $8651 \AA$ and $8736 \AA$ lines present some deviations from linearity at $10^{13} \mathrm{~cm}^{-3}$ due to the Debye cut-off.

\subsubsection{Summary of the discussion}

Considering the validity conditions discussed above, our data can then be used for astrophysical purposes as follows: 
1. If the impact approximation criterion $\left(C_{1} \ll 1\right)$ is satisfied, the resulting profiles are lorentzian, and the total widths and shifts are given by:

$$
W=W_{\mathrm{e}}+W_{\mathrm{H}^{+}}+W_{\mathrm{He}^{+}}+W_{\mathrm{He}^{++}},
$$$$
d=d_{\mathrm{e}}+d_{\mathrm{H}^{+}}+d_{\mathrm{He}^{+}}+d_{\mathrm{He}^{++}} \text {. }
$$

2. If the impact approximation criterion is satisfied for electronic collisions and not satisfied for the collisions with ions, then the quasistatic approximation can be used:

2a. If $C_{2}>1$, the quasistatic interaction is dipolar. This case is not treated in this paper.

$2 \mathrm{~b}$. If the quasistatic interaction is quadratic $\left(C_{2}<1\right)$, which is the predominant case, the resulting widths and shifts are given by:

$$
\begin{aligned}
& W=W_{\mathrm{e}}+W_{\mathrm{iq}}, \\
& d=d_{\mathrm{e}}+d_{\mathrm{iq}} .
\end{aligned}
$$

However, it must be noticed that an intermediate region between the impact and quasistatic approximations is expected for collisions with ions, for which neither of the two limiting approximations is valid. For such conditions the static model employed can only give an estimate for the line shape.

\section{Conclusion}

We have calculated Stark widths and shifts for six infrared lines of helium arising from highly excited levels. We have used the Sahal-Bréchot theory and code which is well adapted to these calculations. We have provided data in the impact and in the quasistatic quadratic approximation. These data can be used for modellisation of early-type stars.
Acknowledgements. This work has been supported by the Cooperation Program "Elargissement des raies spectrales dans un plasma. Application Astrophysique" between the French "Direction des Relations Internationales du CNRS" and the Tunisian DGRST (Direction Générale de la Recherche Scientifique Tunisienne).

\section{References}

Baranger M., 1958a, Phys. Rev. 111, 481

Baranger M., 1958b, Phys. Rev. 111, 494

Baranger M., 1958c, Phys. Rev. 112, 855

Baranger M., 1962, in: Bates D.R. (ed.) Atomic and Molecular Processes. Academic Press, New York

Ben Nessib N., Ben Lakhdar Z., Sahal-Bréchot S., 1996, Phys. Scr. 54, 608

Cunto W., Mendoza C., Ochsenbein F., Zeippen C., 1993, J. Astron. Astrophys. 275, L5

Deutsch C., 1969, Thesis, Université de Paris

Frémat Y., Houziaux L., Andrillat Y., 1996, MNRAS 279, 25

Jaschek M., Andrillat Y., Houziaux L., Jaschek C., 1994, A\&A 282,911

Dimitrijević M.S., Sahal-Bréchot S., 1984, JQSRT 31, 301

Griem H.R., 1974, Plasma Spectroscopy. McGraw-Hill, New York

Griem H.R., 1964, Plasma Spectroscopy. McGraw-Hill, New York

Martin W.C., 1973, J. Phys. Chem. Ref. Data 2, 262

Sahal-Bréchot S., 1969, A\&A 1, 91

Sahal-Bréchot S., 1969, A\&A 2, 322

Traving G., 1968, in: Lochte-Holtgreven W. (ed.) Plasma Diagnostics. North Holland, Amsterdam

Oertel G.K., Shomo L.P., 1968, ApJS 16, 175

Van Regemorter H., Hoang-Binh D., Prudhomme M., 1979, J. Phys. B. 12, 1073 
Table 1. Present calculations for the transition $7{ }^{1} \mathrm{D}-3^{1} \mathrm{P}$ of Helium $(\lambda=10138 \AA)-T$ : electronic temperature in $10^{4} \mathrm{~K}$ $N_{\mathrm{e}}$ : electronic density in $\mathrm{cm}^{-3}$. - $W$ and $d$ : impact half-width and shift (positive shifts are towards the red) in $\AA$ - $A$ : quasistatic quadratic broadening parameter $-C_{1}$ : impact approximation validity criterion (not mentioned if $C_{1}<0.1$ ) - $C_{2}$ : ratio of inelastic collisions contribution to the elastic collisions one to the impact width - $C_{3}$ : one state approximation validity criterion (ratio of the contribution of the upper level to that the lower level to the impact width, not mentioned if $\left.C_{3}>1000\right)-C_{4}$ : perturbation theory validity criterion (contribution of strong collisions to the impact width) - $C_{5}$ : isolated line approximation validity criterion

\begin{tabular}{|c|c|c|c|c|c|c|c|c|c|c|c|}
\hline & \multirow[b]{2}{*}{$\begin{array}{l}T \\
N_{\mathrm{e}}\end{array}$} & \multirow{2}{*}{ 1a: } & \multirow{2}{*}{$\begin{array}{c}\text { Collisions } \\
2\end{array}$} & \multirow{2}{*}{$\begin{aligned} & \text { with } \\
4 & \end{aligned}$} & \multicolumn{2}{|c|}{ electrons } & \multirow{2}{*}{$\frac{1 b:}{1}$} & Collisions & \multirow[t]{2}{*}{ with } & \multicolumn{2}{|c|}{ protons } \\
\hline & & & & & 8 & 10 & & 2 & & 8 & 10 \\
\hline$W$ & $\begin{array}{l}10^{10} \\
10^{11} \\
10^{12} \\
10^{13}\end{array}$ & $\begin{array}{l}1.97 \mathrm{E}-4 \\
1.97 \mathrm{E}-3 \\
1.97 \mathrm{E}-2 \\
0.189\end{array}$ & $\begin{array}{l}1.65 \mathrm{E}-4 \\
1.65 \mathrm{E}-3 \\
1.65 \mathrm{E}-2 \\
0.160\end{array}$ & $\begin{array}{l}1.36 \mathrm{E}-4 \\
1.36 \mathrm{E}-3 \\
1.36 \mathrm{E}-2 \\
0.132\end{array}$ & $\begin{array}{l}1.11 \mathrm{E}-4 \\
1.11 \mathrm{E}-3 \\
1.11 \mathrm{E}-2 \\
0.108\end{array}$ & $\begin{array}{l}1.03 \mathrm{E}-4 \\
1.03 \mathrm{E}-3 \\
1.03 \mathrm{E}-2 \\
0.101\end{array}$ & $\begin{array}{l}2.50 \mathrm{E}-4 \\
2.50 \mathrm{E}-3 \\
2.50 \mathrm{E}-2 \\
0.250\end{array}$ & $\begin{array}{l}2.89 \mathrm{E}-4 \\
2.89 \mathrm{E}-3 \\
2.89 \mathrm{E}-2 \\
0.289\end{array}$ & $\begin{array}{l}3.18 \mathrm{E}-4 \\
3.18 \mathrm{E}-3 \\
3.18 \mathrm{E}-2 \\
0.318\end{array}$ & $\begin{array}{l}3.37 \mathrm{E}-4 \\
3.37 \mathrm{E}-3 \\
3.37 \mathrm{E}-2 \\
0.337\end{array}$ & $\begin{array}{l}3.41 \mathrm{E}-4 \\
3.41 \mathrm{E}-3 \\
3.41 \mathrm{E}-2 \\
0.341\end{array}$ \\
\hline$d$ & $\begin{array}{l}10^{10} \\
10^{11} \\
10^{12} \\
10^{13}\end{array}$ & $\begin{array}{l}2.26 \mathrm{E}-5 \\
2.26 \mathrm{E}-4 \\
2.26 \mathrm{E}-3 \\
2.26 \mathrm{E}-2\end{array}$ & $\begin{array}{l}1.25 \mathrm{E}-5 \\
1.25 \mathrm{E}-4 \\
1.25 \mathrm{E}-3 \\
1.25 \mathrm{E}-2\end{array}$ & $\begin{array}{l}6.26 \mathrm{E}-6 \\
6.26 \mathrm{E}-5 \\
6.26 \mathrm{E}-4 \\
6.26 \mathrm{E}-3\end{array}$ & $\begin{array}{l}2.72 \mathrm{E}-6 \\
2.72 \mathrm{E}-5 \\
2.72 \mathrm{E}-4 \\
2.72 \mathrm{E}-3\end{array}$ & $\begin{array}{l}2.10 \mathrm{E}-6 \\
2.10 \mathrm{E}-5 \\
2.10 \mathrm{E}-4 \\
2.10 \mathrm{E}-3\end{array}$ & $\begin{array}{l}1.79 \mathrm{E}-4 \\
1.79 \mathrm{E}-3 \\
1.76 \mathrm{E}-2 \\
0.167\end{array}$ & $\begin{array}{l}2.05 \mathrm{E}-4 \\
2.04 \mathrm{E}-3 \\
2.03 \mathrm{E}-2 \\
0.197\end{array}$ & $\begin{array}{l}2.20 \mathrm{E}-4 \\
2.20 \mathrm{E}-3 \\
2.18 \mathrm{E}-2 \\
0.214\end{array}$ & $\begin{array}{l}2.18 \mathrm{E}-4 \\
2.18 \mathrm{E}-3 \\
2.18 \mathrm{E}-2 \\
0.215\end{array}$ & $\begin{array}{l}2.12 \mathrm{E}-4 \\
2.12 \mathrm{E}-3 \\
2.12 \mathrm{E}-2 \\
0.210\end{array}$ \\
\hline$A$ & $\begin{array}{l}10^{10} \\
10^{11} \\
10^{12} \\
10^{13}\end{array}$ & & & & & & $\begin{array}{l}0.089 \\
0.159 \\
0.283 \\
0.520\end{array}$ & $\begin{array}{l}0.010 \\
0.182 \\
0.324 \\
0.590\end{array}$ & $\begin{array}{l}0.011 \\
0.210 \\
0.374 \\
0.680\end{array}$ & $\begin{array}{l}0.013 \\
0.246 \\
0.437 \\
0.792\end{array}$ & $\begin{array}{l}0.014 \\
0.259 \\
0.461 \\
0.834\end{array}$ \\
\hline$C_{1}$ & $\begin{array}{l}10^{10} \\
10^{11} \\
10^{12} \\
10^{13}\end{array}$ & $\begin{array}{l}- \\
- \\
- \\
-\end{array}$ & $\begin{array}{l}- \\
- \\
- \\
-\end{array}$ & $\begin{array}{l}- \\
- \\
- \\
-\end{array}$ & $\begin{array}{l}- \\
- \\
- \\
-\end{array}$ & $\begin{array}{l}- \\
- \\
- \\
-\end{array}$ & $\begin{array}{l}- \\
- \\
- \\
0.723\end{array}$ & $\begin{array}{l}- \\
- \\
- \\
0.471\end{array}$ & $\begin{array}{l}- \\
- \\
- \\
0.276\end{array}$ & $\begin{array}{l}- \\
- \\
- \\
0.147\end{array}$ & $\begin{array}{l}- \\
- \\
- \\
0.118\end{array}$ \\
\hline$C_{2}$ & $\begin{array}{l}10^{10} \\
10^{11} \\
10^{12} \\
10^{13}\end{array}$ & & & & & & $\begin{array}{l}0.343 \\
0.343 \\
0.343 \\
0.343\end{array}$ & $\begin{array}{l}0.569 \\
0.569 \\
0.569 \\
0.569\end{array}$ & $\begin{array}{l}0.960 \\
0.960 \\
0.960 \\
0.960\end{array}$ & $\begin{array}{l}1.61 \\
1.61 \\
1.61 \\
1.61\end{array}$ & $\begin{array}{l}1.88 \\
1.88 \\
1.88 \\
1.88\end{array}$ \\
\hline$C_{3}$ & $\begin{array}{l}10^{10} \\
10^{11} \\
10^{12} \\
10^{13}\end{array}$ & $\begin{array}{l}89 \\
89 \\
88 \\
85\end{array}$ & $\begin{array}{l}72 \\
72 \\
78 \\
75\end{array}$ & $\begin{array}{l}69 \\
69 \\
69 \\
66\end{array}$ & $\begin{array}{l}61 \\
61 \\
61 \\
59\end{array}$ & $\begin{array}{l}59 \\
59 \\
59 \\
57\end{array}$ & $\begin{array}{l}- \\
- \\
- \\
-\end{array}$ & $\begin{array}{l}- \\
- \\
-\end{array}$ & $\begin{array}{l}- \\
- \\
-\end{array}$ & $\begin{array}{l}831 \\
831 \\
830 \\
831\end{array}$ & $\begin{array}{l}731 \\
731 \\
730 \\
721\end{array}$ \\
\hline$C_{4}$ & $\begin{array}{l}10^{10} \\
10^{11} \\
10^{12} \\
10^{13}\end{array}$ & $\begin{array}{l}0.136 \\
0.136 \\
0.136 \\
0.142\end{array}$ & $\begin{array}{l}0.121 \\
0.121 \\
0.121 \\
0.125\end{array}$ & $\begin{array}{l}0.111 \\
0.111 \\
0.112 \\
0.115\end{array}$ & $\begin{array}{l}0.105 \\
0.105 \\
0.105 \\
0.108\end{array}$ & $\begin{array}{l}0.104 \\
0.104 \\
0.104 \\
0.106\end{array}$ & $\begin{array}{l}0.619 \\
0.619 \\
0.619 \\
0.619\end{array}$ & $\begin{array}{l}0.568 \\
0.568 \\
0.568 \\
0.568\end{array}$ & $\begin{array}{l}0.512 \\
0.512 \\
0.512 \\
0.512\end{array}$ & $\begin{array}{l}0.447 \\
0.447 \\
0.447 \\
0.447\end{array}$ & $\begin{array}{l}0.428 \\
0.428 \\
0.428 \\
0.428\end{array}$ \\
\hline
\end{tabular}

1c: Isolated line approximation validity criterion

\begin{tabular}{lllllll}
\cline { 3 - 7 }$C_{5}$ & $10^{10}$ & $39.05 \mathrm{E}-5$ & $39.04 \mathrm{E}-5$ & $39.10 \mathrm{E}-5$ & $38.32 \mathrm{E}-5$ & $38.02 \mathrm{E}-5$ \\
& $10^{11}$ & $39.08 \mathrm{E}-4$ & $39.36 \mathrm{E}-4$ & $39.10 \mathrm{E}-4$ & $38.32 \mathrm{E}-4$ & $38.02 \mathrm{E}-4$ \\
& $10^{12}$ & $39.11 \mathrm{E}-3$ & $39.36 \mathrm{E}-3$ & $39.05 \mathrm{E}-3$ & $38.32 \mathrm{E}-3$ & $38.02 \mathrm{E}-3$ \\
& $10^{13}$ & 0.382 & 0.387 & 0.385 & 0.379 & 0.375
\end{tabular}


Table 2. Same as Table 1

\begin{tabular}{|c|c|c|c|c|c|c|c|c|c|c|c|}
\hline & \multirow[b]{2}{*}{$\begin{array}{l}T \\
N_{\mathrm{e}}\end{array}$} & $2 a:$ & Collisions & with & \multicolumn{2}{|c|}{$\mathbf{H e}^{+}$} & $2 b:$ & Collisions & with & \multicolumn{2}{|c|}{$\mathbf{H e}^{++}$} \\
\hline & & 1 & 2 & 4 & 8 & 10 & 1 & 2 & 4 & 8 & 10 \\
\hline$W$ & $\begin{array}{l}10^{10} \\
10^{11} \\
10^{12} \\
10^{13}\end{array}$ & $\begin{array}{l}1.92 \mathrm{E}-4 \\
1.92 \mathrm{E}-3 \\
1.92 \mathrm{E}-2 \\
0.192\end{array}$ & $\begin{array}{l}2.53 \mathrm{E}-4 \\
2.35 \mathrm{E}-3 \\
2.35 \mathrm{E}-2 \\
0.235\end{array}$ & $\begin{array}{l}2.77 \mathrm{E}-4 \\
2.77 \mathrm{E}-3 \\
2.77 \mathrm{E}-2 \\
0.277\end{array}$ & $\begin{array}{l}3.09 \mathrm{E}-4 \\
3.09 \mathrm{E}-3 \\
3.09 \mathrm{E}-2 \\
0.309\end{array}$ & $\begin{array}{l}3.18 \mathrm{E}-4 \\
3.18 \mathrm{E}-3 \\
3.18 \mathrm{E}-2 \\
0.318\end{array}$ & $\begin{array}{l}4.43 \mathrm{E}-4 \\
4.43 \mathrm{E}-3 \\
4.43 \mathrm{E}-2 \\
0.443\end{array}$ & $\begin{array}{l}5.43 \mathrm{E}-4 \\
5.43 \mathrm{E}-3 \\
5.43 \mathrm{E}-2 \\
0.543\end{array}$ & $\begin{array}{l}6.66 \mathrm{E}-4 \\
6.66 \mathrm{E}-3 \\
6.66 \mathrm{E}-2 \\
0.666\end{array}$ & $\begin{array}{l}7.84 \mathrm{E}-4 \\
7.84 \mathrm{E}-3 \\
7.84 \mathrm{E}-2 \\
0.784\end{array}$ & $\begin{array}{l}8.17 \mathrm{E}-4 \\
8.17 \mathrm{E}-3 \\
8.17 \mathrm{E}-2 \\
0.817\end{array}$ \\
\hline$d$ & $\begin{array}{l}10^{10} \\
10^{11} \\
10^{12} \\
10^{13}\end{array}$ & $\begin{array}{l}1.48 \mathrm{E}-4 \\
1.47 \mathrm{E}-3 \\
1.44 \mathrm{E}-2 \\
0.136\end{array}$ & $\begin{array}{l}1.71 \mathrm{E}-4 \\
1.70 \mathrm{E}-3 \\
1.69 \mathrm{E}-2 \\
0.163\end{array}$ & $\begin{array}{l}1.97 \mathrm{E}-4 \\
1.97 \mathrm{E}-3 \\
1.96 \mathrm{E}-2 \\
0.191\end{array}$ & $\begin{array}{l}2.17 \mathrm{E}-4 \\
2.17 \mathrm{E}-3 \\
2.16 \mathrm{E}-2 \\
0.213\end{array}$ & $\begin{array}{l}2.20 \mathrm{E}-4 \\
2.20 \mathrm{E}-3 \\
2.19 \mathrm{E}-2 \\
0.216\end{array}$ & $\begin{array}{l}3.68 \mathrm{E}-4 \\
3.65 \mathrm{E}-3 \\
3.54 \mathrm{E}-2 \\
0.320\end{array}$ & $\begin{array}{l}4.18 \mathrm{E}-4 \\
4.16 \mathrm{E}-3 \\
4.08 \mathrm{E}-2 \\
0.385\end{array}$ & $\begin{array}{l}4.43 \mathrm{E}-4 \\
4.82 \mathrm{E}-3 \\
4.77 \mathrm{E}-2 \\
0.460\end{array}$ & $\begin{array}{l}5.58 \mathrm{E}-4 \\
5.57 \mathrm{E}-3 \\
5.54 \mathrm{E}-2 \\
0.542\end{array}$ & $\begin{array}{l}5.79 \mathrm{E}-4 \\
5.79 \mathrm{E}-3 \\
5.65 \mathrm{E}-2 \\
0.576\end{array}$ \\
\hline$A$ & $\begin{array}{l}10^{10} \\
10^{11} \\
10^{12} \\
10^{13}\end{array}$ & $\begin{array}{l}0.089 \\
0.159 \\
0.283 \\
0.520\end{array}$ & $\begin{array}{l}0.010 \\
0.182 \\
0.324 \\
0.590\end{array}$ & $\begin{array}{l}0.011 \\
0.210 \\
0.374 \\
0.680\end{array}$ & $\begin{array}{l}0.013 \\
0.246 \\
0.437 \\
0.792\end{array}$ & $\begin{array}{l}0.014 \\
0.259 \\
0.461 \\
0.834\end{array}$ & $\begin{array}{l}0.089 \\
0.159 \\
0.283 \\
0.520\end{array}$ & $\begin{array}{l}0.010 \\
0.182 \\
0.324 \\
0.590\end{array}$ & $\begin{array}{l}0.011 \\
0.210 \\
0.374 \\
0.680\end{array}$ & $\begin{array}{l}0.013 \\
0.246 \\
0.437 \\
0.792\end{array}$ & $\begin{array}{l}0.014 \\
0.259 \\
0.461 \\
0.834\end{array}$ \\
\hline$C_{1}$ & $\begin{array}{l}10^{10} \\
10^{11} \\
10^{12} \\
10^{13}\end{array}$ & $\begin{array}{l}- \\
- \\
0.105 \\
1.05\end{array}$ & $\begin{array}{l}- \\
- \\
- \\
0.807\end{array}$ & $\begin{array}{l}- \\
- \\
- \\
0.548\end{array}$ & $\begin{array}{l}- \\
- \\
- \\
0.331\end{array}$ & $\begin{array}{l}- \\
- \\
- \\
0.276\end{array}$ & $\begin{array}{l}- \\
- \\
0.372 \\
3.72\end{array}$ & $\begin{array}{l}- \\
- \\
0.298 \\
2.98\end{array}$ & $\begin{array}{l}- \\
- \\
0.228 \\
2.28\end{array}$ & $\begin{array}{l}- \\
- \\
0.155 \\
1.55\end{array}$ & $\begin{array}{l}- \\
- \\
0.133 \\
1.33\end{array}$ \\
\hline$C_{2}$ & $\begin{array}{l}10^{10} \\
10^{11} \\
10^{12} \\
10^{13}\end{array}$ & $\begin{array}{l}0.177 \\
0.177 \\
0.177 \\
0.177\end{array}$ & $\begin{array}{l}0.291 \\
0.291 \\
0.291 \\
0.291\end{array}$ & $\begin{array}{l}0.481 \\
0.481 \\
0.481 \\
0.481\end{array}$ & $\begin{array}{l}0.814 \\
0.814 \\
0.814 \\
0.814\end{array}$ & $\begin{array}{l}0.966 \\
0.966 \\
0.966 \\
0.966\end{array}$ & $\begin{array}{l}0.105 \\
0.105 \\
0.105 \\
0.105\end{array}$ & $\begin{array}{l}0.177 \\
0.177 \\
0.177 \\
0.177\end{array}$ & $\begin{array}{l}0.292 \\
0.292 \\
0.292 \\
0.292\end{array}$ & $\begin{array}{l}0.481 \\
0.481 \\
0.481 \\
0.481\end{array}$ & $\begin{array}{l}0.569 \\
0.569 \\
0.569 \\
0.569\end{array}$ \\
\hline$C_{3}$ & $\begin{array}{l}10^{10} \\
10^{11} \\
10^{12} \\
10^{13}\end{array}$ & $\begin{array}{l}- \\
- \\
- \\
-\end{array}$ & $\begin{array}{l}- \\
- \\
-\end{array}$ & $\begin{array}{l}- \\
- \\
-\end{array}$ & $\begin{array}{l}- \\
- \\
- \\
-\end{array}$ & $\begin{array}{l}- \\
- \\
-\end{array}$ & $\begin{array}{l}- \\
- \\
-\end{array}$ & $\begin{array}{l}- \\
- \\
-\end{array}$ & $\begin{array}{l}- \\
- \\
-\end{array}$ & $\begin{array}{l}- \\
- \\
- \\
-\end{array}$ & $\begin{array}{l}- \\
- \\
-\end{array}$ \\
\hline$C_{4}$ & $\begin{array}{l}10^{10} \\
10^{11} \\
10^{12} \\
10^{13}\end{array}$ & $\begin{array}{l}0.653 \\
0.653 \\
0.653 \\
0.653\end{array}$ & $\begin{array}{l}0.631 \\
0.631 \\
0.631 \\
0.631\end{array}$ & $\begin{array}{l}0.586 \\
0.586 \\
0.586 \\
0.586\end{array}$ & $\begin{array}{l}0.530 \\
0.530 \\
0.530 \\
0.530\end{array}$ & $\begin{array}{l}0.512 \\
0.512 \\
0.512 \\
0.512\end{array}$ & $\begin{array}{l}0.655 \\
0.655 \\
0.655 \\
0.655\end{array}$ & $\begin{array}{l}0.654 \\
0.654 \\
0.654 \\
0.654\end{array}$ & $\begin{array}{l}0.631 \\
0.631 \\
0.631 \\
0.631\end{array}$ & $\begin{array}{l}0.584 \\
0.584 \\
0.584 \\
0.584\end{array}$ & $\begin{array}{l}0.567 \\
0.567 \\
0.567 \\
0.567\end{array}$ \\
\hline
\end{tabular}


Table 3. Same as Table 1 but for transition $9^{3} \mathrm{D}-3^{3} \mathrm{P}$ of Helium $(\lambda=8779 \AA)$

\begin{tabular}{|c|c|c|c|c|c|c|c|c|c|c|c|}
\hline & \multirow[b]{2}{*}{$\begin{array}{l}T \\
N_{\mathrm{e}}\end{array}$} & \multirow{2}{*}{$\frac{3 a:}{1}$} & \multirow{2}{*}{$\begin{array}{c}\text { Collisions } \\
2\end{array}$} & \multirow{2}{*}{$\begin{array}{l}\text { with } \\
4\end{array}$} & \multicolumn{2}{|c|}{ electrons } & \multirow{2}{*}{$\frac{3 \mathrm{~b}:}{} \frac{}{1}$} & \multicolumn{2}{|c|}{ Collisions } & \multicolumn{2}{|c|}{ protons } \\
\hline & & & & & 8 & 10 & & 2 & 4 & 8 & 10 \\
\hline$W$ & $\begin{array}{l}10^{10} \\
10^{11} \\
10^{12} \\
10^{13}\end{array}$ & $\begin{array}{l}4.51 \mathrm{E}-5 \\
4.51 \mathrm{E}-4 \\
4.48 \mathrm{E}-3 \\
4.19 \mathrm{E}-2\end{array}$ & $\begin{array}{l}3.90 \mathrm{E}-5 \\
3.90 \mathrm{E}-4 \\
3.87 \mathrm{E}-3 \\
3.67 \mathrm{E}-2\end{array}$ & $\begin{array}{l}3.36 \mathrm{E}-5 \\
3.36 \mathrm{E}-4 \\
3.34 \mathrm{E}-3 \\
3.20 \mathrm{E}-2\end{array}$ & $\begin{array}{l}2.83 \mathrm{E}-5 \\
2.83 \mathrm{E}-4 \\
2.82 \mathrm{E}-3 \\
2.72 \mathrm{E}-2\end{array}$ & $\begin{array}{l}2.67 \mathrm{E}-5 \\
2.67 \mathrm{E}-4 \\
2.66 \mathrm{E}-3 \\
2.57 \mathrm{E}-2\end{array}$ & $\begin{array}{l}1.03 \mathrm{E}-4 \\
1.03 \mathrm{E}-3 \\
1.03 \mathrm{E}-2 \\
0.103\end{array}$ & $\begin{array}{l}1.06 \mathrm{E}-4 \\
1.06 \mathrm{E}-3 \\
1.06 \mathrm{E}-2 \\
0.106\end{array}$ & $\begin{array}{l}1.08 \mathrm{E}-4 \\
1.08 \mathrm{E}-3 \\
1.08 \mathrm{E}-2 \\
0.108\end{array}$ & $\begin{array}{l}1.11 \mathrm{E}-4 \\
1.11 \mathrm{E}-3 \\
1.11 \mathrm{E}-2 \\
0.111\end{array}$ & $\begin{array}{l}1.11 \mathrm{E}-4 \\
1.11 \mathrm{E}-3 \\
1.11 \mathrm{E}-2 \\
0.111\end{array}$ \\
\hline$d$ & $\begin{array}{l}10^{10} \\
10^{11} \\
10^{12} \\
10^{13}\end{array}$ & $\begin{array}{l}5.88 \mathrm{E}-6 \\
5.88 \mathrm{E}-5 \\
5.88 \mathrm{E}-4 \\
5.59 \mathrm{E}-3\end{array}$ & $\begin{array}{l}4.24 \mathrm{E}-6 \\
4.24 \mathrm{E}-5 \\
4.24 \mathrm{E}-4 \\
4.24 \mathrm{E}-3\end{array}$ & $\begin{array}{l}2.97 \mathrm{E}-6 \\
2.97 \mathrm{E}-5 \\
2.97 \mathrm{E}-4 \\
2.97 \mathrm{E}-3\end{array}$ & $\begin{array}{l}2.07 \mathrm{E}-6 \\
2.07 \mathrm{E}-5 \\
2.07 \mathrm{E}-4 \\
2.07 \mathrm{E}-3\end{array}$ & $\begin{array}{l}1.85 \mathrm{E}-6 \\
1.85 \mathrm{E}-5 \\
1.85 \mathrm{E}-4 \\
1.85 \mathrm{E}-3\end{array}$ & $\begin{array}{l}7.56 \mathrm{E}-5 \\
7.54 \mathrm{E}-4 \\
7.54 \mathrm{E}-3 \\
7.21 \mathrm{E}-2\end{array}$ & $\begin{array}{l}7.64 \mathrm{E}-5 \\
7.64 \mathrm{E}-4 \\
7.64 \mathrm{E}-3 \\
7.43 \mathrm{E}-2\end{array}$ & $\begin{array}{l}6.84 \mathrm{E}-5 \\
6.84 \mathrm{E}-4 \\
6.84 \mathrm{E}-3 \\
6.74 \mathrm{E}-2\end{array}$ & $\begin{array}{l}5.47 \mathrm{E}-5 \\
5.47 \mathrm{E}-4 \\
5.47 \mathrm{E}-3 \\
5.46 \mathrm{E}-2\end{array}$ & $\begin{array}{l}5.08 \mathrm{E}-5 \\
5.08 \mathrm{E}-4 \\
5.08 \mathrm{E}-3 \\
5.07 \mathrm{E}-2\end{array}$ \\
\hline$A$ & $\begin{array}{l}10^{10} \\
10^{11} \\
10^{12} \\
10^{13}\end{array}$ & & & & & & $\begin{array}{l}0.111 \\
0.198 \\
0.354 \\
0.662\end{array}$ & $\begin{array}{l}0.124 \\
0.221 \\
0.394 \\
0.730\end{array}$ & $\begin{array}{l}0.139 \\
0.247 \\
0.441 \\
0.810\end{array}$ & $\begin{array}{l}0.158 \\
0.280 \\
0.500 \\
0.914\end{array}$ & $\begin{array}{l}0.165 \\
0.293 \\
0.523 \\
0.955\end{array}$ \\
\hline$C_{1}$ & $\begin{array}{l}10^{10} \\
10^{11} \\
10^{12} \\
10^{13}\end{array}$ & $\begin{array}{l}- \\
- \\
- \\
-\end{array}$ & $\begin{array}{l}- \\
- \\
-\end{array}$ & $\begin{array}{l}- \\
- \\
- \\
-\end{array}$ & $\begin{array}{l}- \\
- \\
-\end{array}$ & $\begin{array}{l}- \\
- \\
-\end{array}$ & $\begin{array}{l}- \\
- \\
- \\
0.232\end{array}$ & $\begin{array}{l}- \\
- \\
- \\
0.117\end{array}$ & $\begin{array}{l}- \\
- \\
-\end{array}$ & $\begin{array}{l}- \\
- \\
- \\
-\end{array}$ & $\begin{array}{l}- \\
- \\
- \\
-\end{array}$ \\
\hline$C_{2}$ & $\begin{array}{l}10^{10} \\
10^{11} \\
10^{12} \\
10^{13}\end{array}$ & & & & & & $\begin{array}{l}0.774 \\
0.774 \\
0.774 \\
0.774\end{array}$ & $\begin{array}{l}1.57 \\
1.57 \\
1.57 \\
1.57\end{array}$ & $\begin{array}{l}3.47 \\
3.47 \\
3.47 \\
3.47\end{array}$ & $\begin{array}{l}6.76 \\
6.76 \\
6.76 \\
6.76\end{array}$ & $\begin{array}{l}7.90 \\
7.90 \\
7.90 \\
7.90\end{array}$ \\
\hline$C_{3}$ & $\begin{array}{l}10^{10} \\
10^{11} \\
10^{12} \\
10^{13}\end{array}$ & $\begin{array}{l}78 \\
78 \\
77 \\
72\end{array}$ & $\begin{array}{l}59 \\
59 \\
59 \\
55\end{array}$ & $\begin{array}{l}48 \\
48 \\
48 \\
46\end{array}$ & $\begin{array}{l}41 \\
41 \\
41 \\
39\end{array}$ & $\begin{array}{l}39 \\
39 \\
39 \\
38\end{array}$ & $\begin{array}{l}- \\
- \\
-\end{array}$ & $\begin{array}{l}- \\
- \\
-\end{array}$ & $\begin{array}{l}- \\
- \\
-\end{array}$ & $\begin{array}{l}- \\
- \\
- \\
-\end{array}$ & $\begin{array}{l}- \\
- \\
-\end{array}$ \\
\hline$C_{4}$ & $\begin{array}{l}10^{10} \\
10^{11} \\
10^{12} \\
10^{13}\end{array}$ & $\begin{array}{l}0.155 \\
0.155 \\
0.156 \\
0.167\end{array}$ & $\begin{array}{l}0.163 \\
0.163 \\
0.163 \\
0.173\end{array}$ & $\begin{array}{l}0.159 \\
0.159 \\
0.159 \\
0.167\end{array}$ & $\begin{array}{l}0.152 \\
0.152 \\
0.152 \\
0.159\end{array}$ & $\begin{array}{l}0.150 \\
0.150 \\
0.150 \\
0.157\end{array}$ & $\begin{array}{l}0.527 \\
0.527 \\
0.527 \\
0.527\end{array}$ & $\begin{array}{l}0.457 \\
0.457 \\
0.457\end{array}$ & $\begin{array}{l}0.386 \\
0.386 \\
0.386\end{array}$ & $\begin{array}{l}0.330 \\
0.330 \\
0.330\end{array}$ & $\begin{array}{l}0.308 \\
0.308 \\
0.308\end{array}$ \\
\hline
\end{tabular}

3c: Isolated line approximation validity criterion

$\begin{array}{lllllll}C_{5} & 10^{10} & 2.3 \mathrm{E}-4 & 2.2 \mathrm{E}-4 & 2.1 \mathrm{E}-4 & 2.1 \mathrm{E}-4 & 2.1 \mathrm{E}-4 \\ & 10^{11} & 2.3 \mathrm{E}-3 & 2.2 \mathrm{E}-3 & 3.7 \mathrm{E}-3 & 2.1 \mathrm{E}-3 & 2.1 \mathrm{E}-3 \\ & 10^{12} & 2.3 \mathrm{E}-2 & 2.2 \mathrm{E}-2 & 2.2 \mathrm{E}-2 & 2.1 \mathrm{E}-2 & 2.1 \mathrm{E}-2 \\ & 10^{13} & 0.22 & 0.22 & 0.22 & 0.21 & 0.22\end{array}$


Table 4. Same as Table 3

\begin{tabular}{|c|c|c|c|c|c|c|c|c|c|c|c|}
\hline & \multirow[b]{2}{*}{$\begin{array}{l}T \\
N_{\mathrm{e}}\end{array}$} & 4a: & Collisions & with & \multicolumn{2}{|c|}{$\mathbf{H e}^{+}$} & 4b: & Collisions & with & \multicolumn{2}{|c|}{$\mathbf{H e}^{++}$} \\
\hline & & 1 & 2 & 4 & 8 & 10 & 1 & 2 & 4 & 8 & 10 \\
\hline$W$ & $\begin{array}{l}10^{10} \\
10^{11} \\
10^{12} \\
10^{13}\end{array}$ & $\begin{array}{l}8.92 \mathrm{E}-5 \\
8.92 \mathrm{E}-4 \\
8.92 \mathrm{E}-3 \\
8.92 \mathrm{E}-2\end{array}$ & $\begin{array}{l}1.01 \mathrm{E}-4 \\
1.01 \mathrm{E}-3 \\
1.01 \mathrm{E}-2 \\
0.101\end{array}$ & $\begin{array}{l}1.06 \mathrm{E}-4 \\
1.06 \mathrm{E}-3 \\
1.06 \mathrm{E}-2 \\
0.106\end{array}$ & $\begin{array}{l}1.08 \mathrm{E}-4 \\
1.08 \mathrm{E}-3 \\
1.08 \mathrm{E}-2 \\
0.108\end{array}$ & $\begin{array}{l}1.08 \mathrm{E}-4 \\
1.08 \mathrm{E}-3 \\
1.08 \mathrm{E}-2 \\
0.108\end{array}$ & $\begin{array}{l}2.08 \mathrm{E}-4 \\
2.08 \mathrm{E}-3 \\
2.08 \mathrm{E}-2 \\
0.208\end{array}$ & $\begin{array}{l}2.52 \mathrm{E}-4 \\
2.52 \mathrm{E}-3 \\
2.52 \mathrm{E}-2 \\
0.252\end{array}$ & $\begin{array}{l}2.85 \mathrm{E}-4 \\
2.85 \mathrm{E}-3 \\
2.85 \mathrm{E}-2 \\
0.285\end{array}$ & $\begin{array}{l}2.99 \mathrm{E}-4 \\
2.99 \mathrm{E}-3 \\
2.99 \mathrm{E}-2 \\
0.299\end{array}$ & $\begin{array}{l}3.01 \mathrm{E}-4 \\
3.01 \mathrm{E}-3 \\
3.01 \mathrm{E}-2 \\
0.301\end{array}$ \\
\hline$d$ & $\begin{array}{l}10^{10} \\
10^{11} \\
10^{12} \\
10^{13}\end{array}$ & $\begin{array}{l}6.40 \mathrm{E}-5 \\
6.38 \mathrm{E}-4 \\
6.29 \mathrm{E}-3 \\
6.04 \mathrm{E}-2\end{array}$ & $\begin{array}{l}7.34 \mathrm{E}-5 \\
7.33 \mathrm{E}-4 \\
7.28 \mathrm{E}-3 \\
7.10 \mathrm{E}-2\end{array}$ & $\begin{array}{l}7.73 \mathrm{E}-5 \\
7.72 \mathrm{E}-4 \\
7.70 \mathrm{E}-3 \\
7.58 \mathrm{E}-2\end{array}$ & $\begin{array}{l}7.19 \mathrm{E}-5 \\
7.19 \mathrm{E}-4 \\
7.18 \mathrm{E}-3 \\
7.13 \mathrm{E}-2\end{array}$ & $\begin{array}{l}6.84 \mathrm{E}-5 \\
6.84 \mathrm{E}-4 \\
6.84 \mathrm{E}-3 \\
6.80 \mathrm{E}-2\end{array}$ & $\begin{array}{l}1.55 \mathrm{E}-4 \\
1.54 \mathrm{E}-3 \\
1.51 \mathrm{E}-2 \\
0.141\end{array}$ & $\begin{array}{l}1.81 \mathrm{E}-4 \\
1.80 \mathrm{E}-3 \\
1.78 \mathrm{E}-2 \\
0.171\end{array}$ & $\begin{array}{l}2.08 \mathrm{E}-4 \\
2.07 \mathrm{E}-3 \\
2.06 \mathrm{E}-2 \\
0.201\end{array}$ & $\begin{array}{l}2.18 \mathrm{E}-4 \\
2.18 \mathrm{E}-3 \\
2.18 \mathrm{E}-2 \\
0.214\end{array}$ & $\begin{array}{l}2.16 \mathrm{E}-4 \\
2.16 \mathrm{E}-3 \\
2.16 \mathrm{E}-2 \\
0.213\end{array}$ \\
\hline$A$ & $\begin{array}{l}10^{10} \\
10^{11} \\
10^{12} \\
10^{13}\end{array}$ & $\begin{array}{l}0.111 \\
0.198 \\
0.354 \\
0.662\end{array}$ & $\begin{array}{l}0.124 \\
0.221 \\
0.394 \\
0.730\end{array}$ & $\begin{array}{l}0.139 \\
0.247 \\
0.441 \\
0.810\end{array}$ & $\begin{array}{l}0.158 \\
0.280 \\
0.500 \\
0.914\end{array}$ & $\begin{array}{l}0.165 \\
0.293 \\
0.523 \\
0.955\end{array}$ & $\begin{array}{l}0.111 \\
0.198 \\
0.354 \\
0.662\end{array}$ & $\begin{array}{l}0.124 \\
0.221 \\
0.394 \\
0.730\end{array}$ & $\begin{array}{l}0.139 \\
0.247 \\
0.441 \\
0.810\end{array}$ & $\begin{array}{l}0.158 \\
0.280 \\
0.500 \\
0.914\end{array}$ & $\begin{array}{l}0.165 \\
0.293 \\
0.523 \\
0.955\end{array}$ \\
\hline$C_{1}$ & $\begin{array}{l}10^{10} \\
10^{11} \\
10^{12} \\
10^{13}\end{array}$ & $\begin{array}{l}- \\
- \\
- \\
0.467\end{array}$ & $\begin{array}{l}- \\
- \\
- \\
0.283\end{array}$ & $\begin{array}{l}- \\
- \\
- \\
0.147\end{array}$ & $\begin{array}{l}- \\
- \\
- \\
-\end{array}$ & $\begin{array}{l}- \\
- \\
- \\
-\end{array}$ & $\begin{array}{l}- \\
- \\
0.182 \\
1.82\end{array}$ & $\begin{array}{l}- \\
- \\
0.132 \\
1.32\end{array}$ & $\begin{array}{l}- \\
- \\
- \\
0.801\end{array}$ & $\begin{array}{l}- \\
- \\
- \\
0.415\end{array}$ & $\begin{array}{l}- \\
- \\
- \\
0.330\end{array}$ \\
\hline$C_{2}$ & $\begin{array}{l}10^{10} \\
10^{11} \\
10^{12} \\
10^{13}\end{array}$ & $\begin{array}{l}0.361 \\
0.361 \\
0.361 \\
0.361\end{array}$ & $\begin{array}{l}0.633 \\
0.633 \\
0.633 \\
0.633\end{array}$ & $\begin{array}{l}1.24 \\
1.24 \\
1.24 \\
1.24\end{array}$ & $\begin{array}{l}2.66 \\
2.66 \\
2.66 \\
2.66\end{array}$ & $\begin{array}{l}3.47 \\
3.47 \\
3.47 \\
3.47\end{array}$ & $\begin{array}{l}0.217 \\
0.217 \\
0.217 \\
0.217\end{array}$ & $\begin{array}{l}0.374 \\
0.374 \\
0.374 \\
0.374\end{array}$ & $\begin{array}{l}0.632 \\
0.632 \\
0.632 \\
0.632\end{array}$ & $\begin{array}{l}1.24 \\
1.24 \\
1.24 \\
1.24\end{array}$ & $\begin{array}{l}1.57 \\
1.57 \\
1.57 \\
1.57\end{array}$ \\
\hline$C_{3}$ & $\begin{array}{l}10^{10} \\
10^{11} \\
10^{12} \\
10^{13}\end{array}$ & $\begin{array}{l}- \\
- \\
- \\
-\end{array}$ & $\begin{array}{l}- \\
- \\
- \\
-\end{array}$ & $\begin{array}{l}- \\
- \\
-\end{array}$ & $\begin{array}{l}- \\
- \\
-\end{array}$ & $\begin{array}{l}- \\
- \\
- \\
-\end{array}$ & $\begin{array}{l}- \\
- \\
- \\
-\end{array}$ & $\begin{array}{l}- \\
- \\
- \\
-\end{array}$ & $\begin{array}{l}- \\
- \\
-\end{array}$ & $\begin{array}{l}- \\
- \\
-\end{array}$ & $\begin{array}{l}- \\
- \\
-\end{array}$ \\
\hline$C_{4}$ & $\begin{array}{l}10^{10} \\
10^{11} \\
10^{12} \\
10^{13}\end{array}$ & $\begin{array}{l}0.614 \\
0.614 \\
0.614 \\
0.614\end{array}$ & $\begin{array}{l}0.550 \\
0.550 \\
0.550 \\
0.550\end{array}$ & $\begin{array}{l}0.478 \\
0.478 \\
0.478 \\
0.478\end{array}$ & $\begin{array}{l}0.406 \\
0.406 \\
0.406 \\
0.406\end{array}$ & $\begin{array}{l}0.386 \\
0.386 \\
0.386 \\
0.386\end{array}$ & $\begin{array}{l}0.605 \\
0.605 \\
0.605 \\
0.605\end{array}$ & $\begin{array}{l}0.612 \\
0.612 \\
0.612 \\
0.612\end{array}$ & $\begin{array}{l}0.549 \\
0.549 \\
0.549 \\
0.549\end{array}$ & $\begin{array}{l}0.478 \\
0.478 \\
0.478 \\
0.478\end{array}$ & $\begin{array}{l}0.456 \\
0.456 \\
0.456 \\
0.456\end{array}$ \\
\hline
\end{tabular}


Table 5. Same as Table 1 but for transition: $13^{3} \mathrm{~F}-3^{3} \mathrm{D}$ of Helium $(\lambda=8651 \AA)$

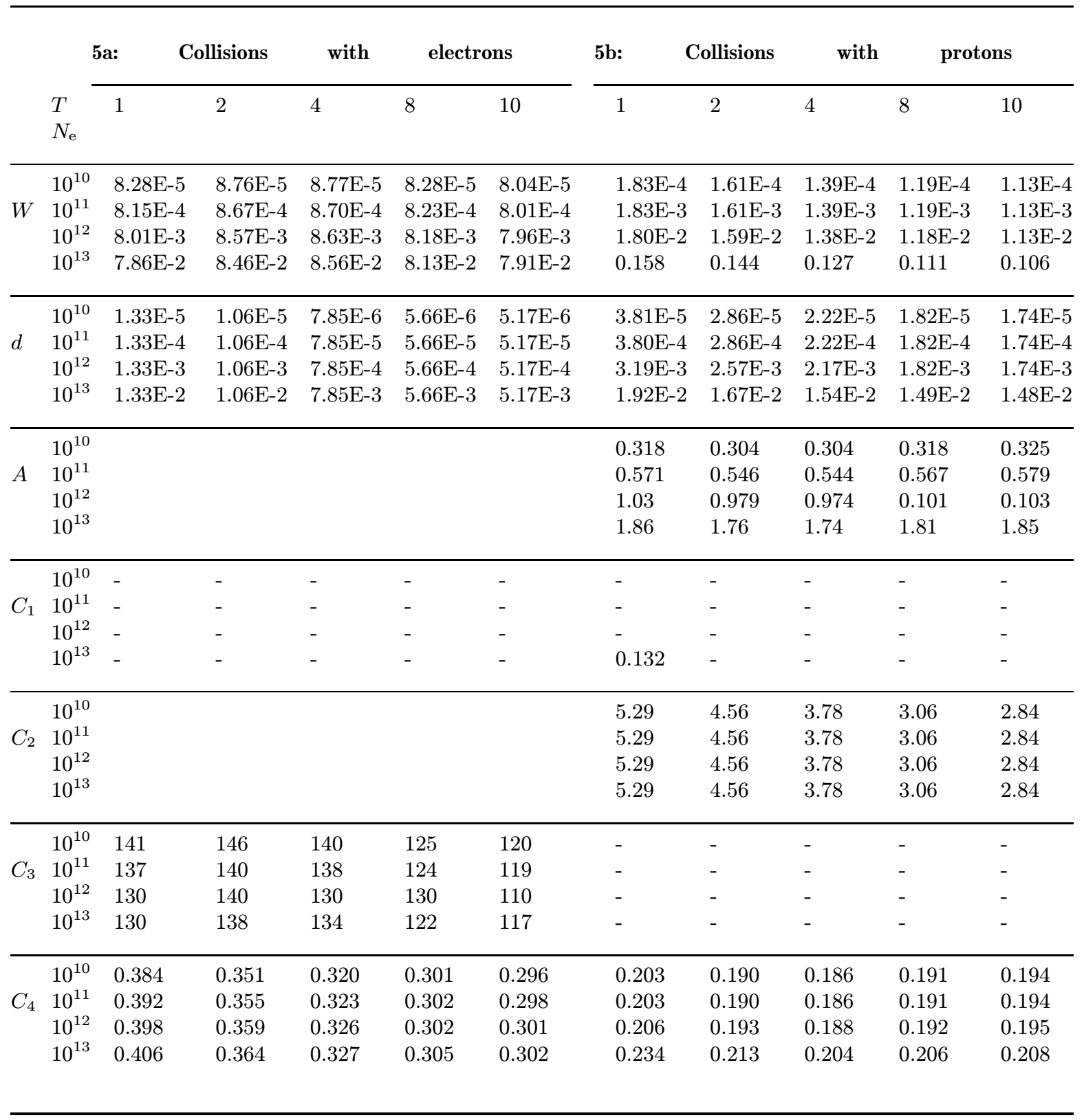

5c: Isolated line approximation validity criterion

\begin{tabular}{cllllll}
\cline { 2 - 7 }$C_{5}$ & $10^{10}$ & $11.1 \mathrm{E}-5$ & $10.4 \mathrm{E}-5$ & $9.5 \mathrm{E}-5$ & $8.5 \mathrm{E}-5$ & $8.2 \mathrm{E}-5$ \\
& $10^{11}$ & $11.0 \mathrm{E}-4$ & $10.4 \mathrm{E}-4$ & $9.5 \mathrm{E}-4$ & $8.5 \mathrm{E}-4$ & $8.2 \mathrm{E}-4$ \\
& $10^{12}$ & $11.0 \mathrm{E}-3$ & $10.3 \mathrm{E}-3$ & $9.4 \mathrm{E}-3$ & $8.4 \mathrm{E}-3$ & $8.1 \mathrm{E}-3$ \\
& $10^{13}$ & $9.9 \mathrm{E}-2$ & $9.6 \mathrm{E}-2$ & $8.9 \mathrm{E}-2$ & $7.8 \mathrm{E}-2$ & $7.8 \mathrm{E}-2$
\end{tabular}


Table 6. Same as Table 5

\begin{tabular}{|c|c|c|c|c|c|c|c|c|c|c|c|}
\hline & \multirow[b]{2}{*}{$\begin{array}{l}T \\
N_{\mathrm{e}}\end{array}$} & 6a: & Collisions & with & \multicolumn{2}{|c|}{$\mathbf{H e}^{+}$} & 6b: & Collisions & with & \multicolumn{2}{|c|}{$\mathbf{H e}^{++}$} \\
\hline & & 1 & 2 & 4 & 8 & 10 & 1 & 2 & 4 & 8 & 10 \\
\hline$W$ & $\begin{array}{l}10^{10} \\
10^{11} \\
10^{12} \\
10^{13}\end{array}$ & $\begin{array}{l}2.07 \mathrm{E}-4 \\
2.07 \mathrm{E}-3 \\
2.06 \mathrm{E}-2 \\
0.187\end{array}$ & $\begin{array}{l}1.89 \mathrm{E}-4 \\
1.89 \mathrm{E}-3 \\
1.89 \mathrm{E}-2 \\
0.176\end{array}$ & $\begin{array}{l}1.68 \mathrm{E}-4 \\
1.68 \mathrm{E}-3 \\
1.68 \mathrm{E}-2 \\
0.159\end{array}$ & $\begin{array}{l}1.46 \mathrm{E}-4 \\
1.46 \mathrm{E}-3 \\
1.46 \mathrm{E}-2 \\
0.139\end{array}$ & $\begin{array}{l}1.39 \mathrm{E}-4 \\
1.39 \mathrm{E}-3 \\
1.39 \mathrm{E}-2 \\
0.133\end{array}$ & $\begin{array}{l}5.99 \mathrm{E}-4 \\
5.99 \mathrm{E}-3 \\
5.95 \mathrm{E}-2 \\
0.521\end{array}$ & $\begin{array}{l}5.63 \mathrm{E}-4 \\
5.63 \mathrm{E}-3 \\
5.60 \mathrm{E}-2 \\
0.508\end{array}$ & $\begin{array}{l}5.13 \mathrm{E}-4 \\
5.13 \mathrm{E}-3 \\
5.11 \mathrm{E}-2 \\
0.474\end{array}$ & $\begin{array}{l}4.54 \mathrm{E}-4 \\
4.54 \mathrm{E}-3 \\
4.53 \mathrm{E}-2 \\
0.427\end{array}$ & $\begin{array}{l}4.34 \mathrm{E}-4 \\
4.34-3 \\
4.33 \mathrm{E}-2 \\
0.410\end{array}$ \\
\hline$d$ & $\begin{array}{l}10^{10} \\
10^{11} \\
10^{12} \\
10^{13}\end{array}$ & $\begin{array}{l}5.71 \mathrm{E}-5 \\
5.69 \mathrm{E}-4 \\
5.05 \mathrm{E}-2 \\
3.31 \mathrm{E}-2\end{array}$ & $\begin{array}{l}4.20 \mathrm{E}-5 \\
4.20 \mathrm{E}-4 \\
3.85 \mathrm{E}-3 \\
2.62 \mathrm{E}-2\end{array}$ & $\begin{array}{l}3.13 \mathrm{E}-5 \\
3.13 \mathrm{E}-4 \\
3.03 \mathrm{E}-3 \\
2.16 \mathrm{E}-2\end{array}$ & $\begin{array}{l}2.40 \mathrm{E}-5 \\
2.40 \mathrm{E}-4 \\
2.39 \mathrm{E}-3 \\
1.87 \mathrm{E}-2\end{array}$ & $\begin{array}{l}2.22 \mathrm{E}-5 \\
2.22 \mathrm{E}-4 \\
2.22 \mathrm{E}-3 \\
1.80 \mathrm{E}-2\end{array}$ & $\begin{array}{l}2.16 \mathrm{E}-4 \\
2.11 \mathrm{E}-3 \\
1.85 \mathrm{E}-2 \\
0.115\end{array}$ & $\begin{array}{l}1.61 \mathrm{E}-4 \\
1.61 \mathrm{E}-3 \\
1.43 \mathrm{E}-2 \\
9.35 \mathrm{E}-2\end{array}$ & $\begin{array}{l}1.19 \mathrm{E}-4 \\
1.19 \mathrm{E}-3 \\
1.09 \mathrm{E}-2 \\
7.40 \mathrm{E}-2\end{array}$ & $\begin{array}{l}8.84 \mathrm{E}-5 \\
8.84 \mathrm{E}-4 \\
8.56 \mathrm{E}-3 \\
6.10 \mathrm{E}-2\end{array}$ & $\begin{array}{l}8.09 \mathrm{E}-5 \\
8.09 \mathrm{E}-4 \\
7.97 \mathrm{E}-3 \\
5.79 \mathrm{E}-2\end{array}$ \\
\hline$A$ & $\begin{array}{l}10^{10} \\
10^{11} \\
10^{12} \\
10^{13}\end{array}$ & $\begin{array}{l}0.318 \\
0.571 \\
1.03 \\
1.86\end{array}$ & $\begin{array}{l}0.304 \\
0.546 \\
0.979 \\
1.76\end{array}$ & $\begin{array}{l}0.304 \\
0.544 \\
0.974 \\
1.74\end{array}$ & $\begin{array}{l}0.318 \\
0.567 \\
0.101 \\
1.81\end{array}$ & $\begin{array}{l}0.325 \\
0.579 \\
0.103 \\
1.85\end{array}$ & $\begin{array}{l}0.318 \\
0.571 \\
1.03 \\
1.86\end{array}$ & $\begin{array}{l}0.304 \\
0.546 \\
0.979 \\
1.76\end{array}$ & $\begin{array}{l}0.304 \\
0.544 \\
0.974 \\
1.74\end{array}$ & $\begin{array}{l}0.318 \\
0.567 \\
0.101 \\
1.81\end{array}$ & $\begin{array}{l}0.325 \\
0.579 \\
0.103 \\
1.85\end{array}$ \\
\hline$C_{1}$ & $\begin{array}{l}10^{10} \\
10^{11} \\
10^{12} \\
10^{13}\end{array}$ & $\begin{array}{l}- \\
- \\
- \\
0.411\end{array}$ & $\begin{array}{l}- \\
- \\
- \\
0.180\end{array}$ & $\begin{array}{l}- \\
- \\
- \\
-\end{array}$ & $\begin{array}{l}- \\
- \\
-\end{array}$ & $\begin{array}{l}- \\
- \\
- \\
-\end{array}$ & $\begin{array}{l}- \\
- \\
0.245 \\
2.45\end{array}$ & $\begin{array}{l}- \\
- \\
0.104 \\
1.04\end{array}$ & $\begin{array}{l}- \\
- \\
- \\
0.442\end{array}$ & $\begin{array}{l}- \\
- \\
- \\
0.190\end{array}$ & $\begin{array}{l}- \\
- \\
- \\
0.146\end{array}$ \\
\hline$C_{2}$ & $\begin{array}{l}10^{10} \\
10^{11} \\
10^{12} \\
10^{13}\end{array}$ & $\begin{array}{l}5.84 \\
5.84 \\
5.84 \\
5.84\end{array}$ & $\begin{array}{l}5.57 \\
5.57 \\
5.57 \\
5.57\end{array}$ & $\begin{array}{l}4.88 \\
4.88 \\
4.88 \\
4.88\end{array}$ & $\begin{array}{l}4.08 \\
4.08 \\
4.08 \\
4.08\end{array}$ & $\begin{array}{l}3.84 \\
3.84 \\
3.84 \\
3.84\end{array}$ & $\begin{array}{l}5.49 \\
5.49 \\
5.49 \\
5.49\end{array}$ & $\begin{array}{l}7.04 \\
7.04 \\
7.04 \\
7.04\end{array}$ & $\begin{array}{l}7.04 \\
7.04 \\
7.04 \\
7.04\end{array}$ & $\begin{array}{l}6.21 \\
6.21 \\
6.21 \\
6.21\end{array}$ & $\begin{array}{l}5.92 \\
5.92 \\
5.92 \\
5.92\end{array}$ \\
\hline$C_{3}$ & $\begin{array}{l}10^{10} \\
10^{11} \\
10^{12} \\
10^{13}\end{array}$ & $\begin{array}{l}- \\
- \\
-\end{array}$ & $\begin{array}{l}- \\
- \\
- \\
-\end{array}$ & $\begin{array}{l}- \\
- \\
- \\
-\end{array}$ & $\begin{array}{l}- \\
- \\
- \\
-\end{array}$ & $\begin{array}{l}- \\
- \\
- \\
-\end{array}$ & $\begin{array}{l}- \\
- \\
-\end{array}$ & $\begin{array}{l}- \\
- \\
- \\
-\end{array}$ & $\begin{array}{l}- \\
- \\
- \\
-\end{array}$ & $\begin{array}{l}- \\
- \\
- \\
-\end{array}$ & $\begin{array}{l}- \\
- \\
-\end{array}$ \\
\hline$C_{4}$ & $\begin{array}{l}10^{10} \\
10^{11} \\
10^{12} \\
10^{13}\end{array}$ & $\begin{array}{l}0.236 \\
0.236 \\
0.237 \\
0.260\end{array}$ & $\begin{array}{l}0.209 \\
0.209 \\
0.210 \\
0.226\end{array}$ & $\begin{array}{l}0.193 \\
0.193 \\
0.194 \\
0.205\end{array}$ & $\begin{array}{l}0.186 \\
0.186 \\
0.187 \\
0.196\end{array}$ & $\begin{array}{l}0.186 \\
0.186 \\
0.187 \\
0.195\end{array}$ & $\begin{array}{l}0.267 \\
0.267 \\
0.269 \\
0.308\end{array}$ & $\begin{array}{l}0.226 \\
0.226 \\
0.228 \\
0.251\end{array}$ & $\begin{array}{l}0.200 \\
0.200 \\
0.200 \\
0.216\end{array}$ & $\begin{array}{l}0.182 \\
0.182 \\
0.182 \\
0.194\end{array}$ & $\begin{array}{l}0.178 \\
0.178 \\
0.178 \\
0.189\end{array}$ \\
\hline
\end{tabular}


Table 7. Same as Table 1 but for transition $10^{3} \mathrm{D}-3^{3} \mathrm{P}$ of Helium $(\lambda=8584 \AA)$

\begin{tabular}{|c|c|c|c|c|c|c|c|c|c|c|c|}
\hline & \multirow[b]{2}{*}{$\begin{array}{l}T \\
N_{\mathrm{e}}\end{array}$} & \multirow{2}{*}{$\frac{7 a:}{1}$} & Collisions & \multirow{2}{*}{$\begin{aligned} \text { with } \\
4\end{aligned}$} & \multicolumn{2}{|c|}{ electrons } & \multirow{2}{*}{$\frac{7 b:}{1}$} & Collisions & \multirow[t]{2}{*}{ with } & \multicolumn{2}{|c|}{ protons } \\
\hline & & & 2 & & 8 & 10 & & 2 & & 8 & 10 \\
\hline$W$ & $\begin{array}{l}10^{10} \\
10^{11} \\
10^{12} \\
10^{13}\end{array}$ & $\begin{array}{l}3.16 \mathrm{E}-5 \\
3.16 \mathrm{E}-4 \\
3.15 \mathrm{E}-3 \\
3.12 \mathrm{E}-2\end{array}$ & $\begin{array}{l}3.34 \mathrm{E}-5 \\
3.34 \mathrm{E}-4 \\
3.34 \mathrm{E}-3 \\
3.32 \mathrm{E}-2\end{array}$ & $\begin{array}{l}3.39 \mathrm{E}-5 \\
3.39 \mathrm{E}-4 \\
3.39 \mathrm{E}-3 \\
3.37 \mathrm{E}-2\end{array}$ & $\begin{array}{l}3.27 \mathrm{E}-5 \\
3.27 \mathrm{E}-4 \\
3.27 \mathrm{E}-3 \\
3.25 \mathrm{E}-2\end{array}$ & $\begin{array}{l}3.20 \mathrm{E}-5 \\
3.20 \mathrm{E}-4 \\
3.20 \mathrm{E}-3 \\
3.19 \mathrm{E}-2\end{array}$ & $\begin{array}{l}3.19 \mathrm{E}-5 \\
3.19 \mathrm{E}-4 \\
3.19 \mathrm{E}-3 \\
3.19 \mathrm{E}-2\end{array}$ & $\begin{array}{l}3.33 \mathrm{E}-5 \\
3.33 \mathrm{E}-4 \\
3.33 \mathrm{E}-3 \\
3.33 \mathrm{E}-2\end{array}$ & $\begin{array}{l}3.34 \mathrm{E}-5 \\
3.34 \mathrm{E}-4 \\
3.34 \mathrm{E}-3 \\
3.34 \mathrm{E}-2\end{array}$ & $\begin{array}{l}3.25 \mathrm{E}-5 \\
3.25 \mathrm{E}-4 \\
3.25 \mathrm{E}-3 \\
3.25 \mathrm{E}-2\end{array}$ & $\begin{array}{l}3.21 \mathrm{E}-5 \\
3.21 \mathrm{E}-4 \\
3.21 \mathrm{E}-3 \\
3.21 \mathrm{E}-2\end{array}$ \\
\hline$d$ & $\begin{array}{l}10^{10} \\
10^{11} \\
10^{12} \\
10^{13}\end{array}$ & $\begin{array}{l}6.56 \mathrm{E}-6 \\
6.56 \mathrm{E}-5 \\
6.56 \mathrm{E}-4 \\
6.52 \mathrm{E}-3\end{array}$ & $\begin{array}{l}5.17 \mathrm{E}-6 \\
5.17 \mathrm{E}-5 \\
5.17 \mathrm{E}-4 \\
5.16 \mathrm{E}-3\end{array}$ & $\begin{array}{l}4.06 \mathrm{E}-6 \\
4.06 \mathrm{E}-5 \\
4.06 \mathrm{E}-4 \\
4.06 \mathrm{E}-3\end{array}$ & $\begin{array}{l}3.00 \mathrm{E}-6 \\
3.00 \mathrm{E}-5 \\
3.00 \mathrm{E}-4 \\
3.00 \mathrm{E}-3\end{array}$ & $\begin{array}{l}2.73 \mathrm{E}-6 \\
2.73 \mathrm{E}-5 \\
2.73 \mathrm{E}-4 \\
2.73 \mathrm{E}-3\end{array}$ & $\begin{array}{l}1.33 \mathrm{E}-5 \\
1.33 \mathrm{E}-4 \\
1.33 \mathrm{E}-3 \\
1.29 \mathrm{E}-2\end{array}$ & $\begin{array}{l}1.12 \mathrm{E}-5 \\
1.12 \mathrm{E}-4 \\
1.12 \mathrm{E}-3 \\
1.10 \mathrm{E}-2\end{array}$ & $\begin{array}{l}9.40 \mathrm{E}-6 \\
9.40 \mathrm{E}-5 \\
9.40 \mathrm{E}-4 \\
9.29 \mathrm{E}-3\end{array}$ & $\begin{array}{l}7.78 \mathrm{E}-6 \\
7.78 \mathrm{E}-5 \\
7.78 \mathrm{E}-4 \\
7.75 \mathrm{E}-3\end{array}$ & $\begin{array}{l}7.37 \mathrm{E}-6 \\
7.37 \mathrm{E}-5 \\
7.37 \mathrm{E}-4 \\
7.36 \mathrm{E}-3\end{array}$ \\
\hline$A$ & $\begin{array}{l}10^{10} \\
10^{11} \\
10^{12} \\
10^{13}\end{array}$ & & & & & & $\begin{array}{l}0.030 \\
0.054 \\
0.097 \\
0.174\end{array}$ & $\begin{array}{l}0.029 \\
0.052 \\
0.092 \\
0.166\end{array}$ & $\begin{array}{l}0.029 \\
0.051 \\
0.091 \\
0.164\end{array}$ & $\begin{array}{l}0.029 \\
0.053 \\
0.094 \\
0.169\end{array}$ & $\begin{array}{l}0.030 \\
0.054 \\
0.096 \\
0.171\end{array}$ \\
\hline$C_{1}$ & $\begin{array}{l}10^{10} \\
10^{11} \\
10^{12} \\
10^{13}\end{array}$ & $\begin{array}{l}- \\
- \\
- \\
-\end{array}$ & $\begin{array}{l}- \\
- \\
- \\
-\end{array}$ & $\begin{array}{l}- \\
- \\
- \\
-\end{array}$ & $\begin{array}{l}- \\
- \\
- \\
-\end{array}$ & $\begin{array}{l}- \\
- \\
- \\
-\end{array}$ & $\begin{array}{l}- \\
- \\
- \\
-\end{array}$ & $\begin{array}{l}- \\
- \\
- \\
-\end{array}$ & $\begin{array}{l}- \\
- \\
- \\
-\end{array}$ & $\begin{array}{l}- \\
- \\
- \\
-\end{array}$ & $\begin{array}{l}- \\
- \\
-\end{array}$ \\
\hline$C_{2}$ & $\begin{array}{l}10^{10} \\
10^{11} \\
10^{12} \\
10^{13}\end{array}$ & & & & & & $\begin{array}{l}1.04 \\
1.04 \\
1.04 \\
1.04\end{array}$ & $\begin{array}{l}1.23 \\
1.23 \\
1.23 \\
1.23\end{array}$ & $\begin{array}{l}1.31 \\
1.31 \\
1.31 \\
1.31\end{array}$ & $\begin{array}{l}1.29 \\
1.29 \\
1.29 \\
1.29\end{array}$ & $\begin{array}{l}1.27 \\
1.27 \\
1.27 \\
1.27\end{array}$ \\
\hline$C_{3}$ & $\begin{array}{l}10^{10} \\
10^{11} \\
10^{12} \\
10^{13}\end{array}$ & $\begin{array}{l}32 \\
32 \\
32 \\
31\end{array}$ & $\begin{array}{l}31 \\
31 \\
31 \\
31\end{array}$ & $\begin{array}{l}31 \\
31 \\
30 \\
30\end{array}$ & $\begin{array}{l}29 \\
29 \\
29 \\
29\end{array}$ & $\begin{array}{l}29 \\
29 \\
28 \\
28\end{array}$ & $\begin{array}{l}- \\
- \\
- \\
-\end{array}$ & $\begin{array}{l}- \\
- \\
- \\
-\end{array}$ & $\begin{array}{l}- \\
- \\
- \\
-\end{array}$ & $\begin{array}{l}- \\
- \\
- \\
-\end{array}$ & $\begin{array}{l}995 \\
995 \\
995 \\
995\end{array}$ \\
\hline$C_{4}$ & $\begin{array}{l}10^{10} \\
10^{11} \\
10^{12} \\
10^{13}\end{array}$ & $\begin{array}{l}0.400 \\
0.400 \\
0.400 \\
0.406\end{array}$ & $\begin{array}{l}0.377 \\
0.377 \\
0.377 \\
0.380\end{array}$ & $\begin{array}{l}0.350 \\
0.350 \\
0.350 \\
0.352\end{array}$ & $\begin{array}{l}0.332 \\
0.332 \\
0.332 \\
0.333\end{array}$ & $\begin{array}{l}0.328 \\
0.328 \\
0.328 \\
0.329\end{array}$ & $\begin{array}{l}0.422 \\
0.422 \\
0.422 \\
0.422\end{array}$ & $\begin{array}{l}0.375 \\
0.375 \\
0.375 \\
0.375\end{array}$ & $\begin{array}{l}0.339 \\
0.339 \\
0.339 \\
0.339\end{array}$ & $\begin{array}{l}0.320 \\
0.320 \\
0.320 \\
0.320\end{array}$ & $\begin{array}{l}0.318 \\
0.318 \\
0.318 \\
0.318\end{array}$ \\
\hline
\end{tabular}

7c: Isolated line approximation validity criterion

\begin{tabular}{lllllll}
\cline { 2 - 7 }$C_{5}$ & $10^{10}$ & $14.8 \mathrm{E}-5$ & $15.6 \mathrm{E}-5$ & $15.8 \mathrm{E}-5$ & $15.2 \mathrm{E}-5$ & $15.0 \mathrm{E}-5$ \\
& $10^{11}$ & $14.8 \mathrm{E}-4$ & $15.6 \mathrm{E}-4$ & $15.8 \mathrm{E}-4$ & $15.2 \mathrm{E}-4$ & $15.0 \mathrm{E}-4$ \\
& $10^{12}$ & $14.8 \mathrm{E}-3$ & $15.6 \mathrm{E}-3$ & $15.8 \mathrm{E}-3$ & $15.2 \mathrm{E}-3$ & $15.0 \mathrm{E}-3$ \\
& $10^{13}$ & 0.147 & 0.155 & 0.157 & 0.152 & 0.150
\end{tabular}


Table 8. Same as Table 7

\begin{tabular}{|c|c|c|c|c|c|c|c|c|c|c|c|}
\hline & \multirow[b]{2}{*}{$\begin{array}{l}T \\
N_{\mathrm{e}}\end{array}$} & 8a: & Collisions & with & \multicolumn{2}{|c|}{$\mathbf{H e}^{+}$} & \multirow{2}{*}{$\frac{8 b:}{1}$} & Collisions & with & \multicolumn{2}{|c|}{$\mathbf{H e}^{++}$} \\
\hline & & 1 & 2 & 4 & 8 & 10 & & 2 & 4 & 8 & 10 \\
\hline$W$ & $\begin{array}{l}10^{10} \\
10^{11} \\
10^{12} \\
10^{13}\end{array}$ & $\begin{array}{l}2.88 \mathrm{E}-5 \\
2.88 \mathrm{E}-4 \\
2.88 \mathrm{E}-3 \\
2.88 \mathrm{E}-2\end{array}$ & $\begin{array}{l}3.12 \mathrm{E}-5 \\
3.12 \mathrm{E}-4 \\
3.12 \mathrm{E}-3 \\
3.12 \mathrm{E}-2\end{array}$ & $\begin{array}{l}3.30 \mathrm{E}-5 \\
3.30 \mathrm{E}-4 \\
3.30 \mathrm{E}-3 \\
3.30 \mathrm{E}-2\end{array}$ & $\begin{array}{l}3.35 \mathrm{E}-5 \\
3.35 \mathrm{E}-4 \\
3.35 \mathrm{E}-3 \\
3.35 \mathrm{E}-2\end{array}$ & $\begin{array}{l}3.34 \mathrm{E}-5 \\
3.34 \mathrm{E}-4 \\
3.34 \mathrm{E}-3 \\
3.34 \mathrm{E}-2\end{array}$ & $\begin{array}{l}6.58 \mathrm{E}-5 \\
6.58 \mathrm{E}-4 \\
6.58 \mathrm{E}-3 \\
6.58 \mathrm{E}-2\end{array}$ & $\begin{array}{l}7.26 \mathrm{E}-5 \\
7.26 \mathrm{E}-4 \\
7.26 \mathrm{E}-3 \\
7.26 \mathrm{E}-2\end{array}$ & $\begin{array}{l}7.83 \mathrm{E}-5 \\
7.83 \mathrm{E}-4 \\
7.83 \mathrm{E}-3 \\
7.83 \mathrm{E}-2\end{array}$ & $\begin{array}{l}8.27 \mathrm{E}-5 \\
8.27 \mathrm{E}-4 \\
8.27 \mathrm{E}-3 \\
8.27 \mathrm{E}-2\end{array}$ & $\begin{array}{l}8.36 \mathrm{E}-5 \\
8.35 \mathrm{E}-4 \\
8.35 \mathrm{E}-3 \\
8.35 \mathrm{E}-2\end{array}$ \\
\hline$d$ & $\begin{array}{l}10^{10} \\
10^{11} \\
10^{12} \\
10^{13}\end{array}$ & $\begin{array}{l}1.49 \mathrm{E}-5 \\
1.49 \mathrm{E}-4 \\
1.48 \mathrm{E}-3 \\
1.45 \mathrm{E}-2\end{array}$ & $\begin{array}{l}1.39 \mathrm{E}-5 \\
1.39 \mathrm{E}-4 \\
1.39 \mathrm{E}-3 \\
1.37 \mathrm{E}-2\end{array}$ & $\begin{array}{l}1.19 \mathrm{E}-5 \\
1.19 \mathrm{E}-4 \\
1.19 \mathrm{E}-3 \\
1.18 \mathrm{E}-2\end{array}$ & $\begin{array}{l}9.98 \mathrm{E}-6 \\
9.98 \mathrm{E}-5 \\
9.98 \mathrm{E}-4 \\
9.94 \mathrm{E}-3\end{array}$ & $\begin{array}{l}9.40 \mathrm{E}-6 \\
9.40 \mathrm{E}-5 \\
9.40 \mathrm{E}-4 \\
9.38 \mathrm{E}-3\end{array}$ & $\begin{array}{l}4.04 \mathrm{E}-5 \\
4.03 \mathrm{E}-4 \\
3.99 \mathrm{E}-3 \\
3.86 \mathrm{E}-2\end{array}$ & $\begin{array}{l}4.21 \mathrm{E}-5 \\
4.20 \mathrm{E}-4 \\
4.18 \mathrm{E}-3 \\
4.09 \mathrm{E}-2\end{array}$ & $\begin{array}{l}3.94 \mathrm{E}-5 \\
3.94 \mathrm{E}-4 \\
3.93 \mathrm{E}-3 \\
3.87 \mathrm{E}-2\end{array}$ & $\begin{array}{l}3.36 \mathrm{E}-5 \\
3.36 \mathrm{E}-4 \\
3.36 \mathrm{E}-3 \\
3.33 \mathrm{E}-2\end{array}$ & $\begin{array}{l}3.18 \mathrm{E}-5 \\
3.18 \mathrm{E}-4 \\
3.18 \mathrm{E}-3 \\
3.15 \mathrm{E}-2\end{array}$ \\
\hline$A$ & $\begin{array}{l}10^{10} \\
10^{11} \\
10^{12} \\
10^{13}\end{array}$ & $\begin{array}{l}0.030 \\
0.054 \\
0.097 \\
0.174\end{array}$ & $\begin{array}{l}0.029 \\
0.052 \\
0.092 \\
0.166\end{array}$ & $\begin{array}{l}0.029 \\
0.051 \\
0.091 \\
0.164\end{array}$ & $\begin{array}{l}0.029 \\
0.053 \\
0.094 \\
0.169\end{array}$ & $\begin{array}{l}0.030 \\
0.054 \\
0.096 \\
0.171\end{array}$ & $\begin{array}{l}0.030 \\
0.054 \\
0.097 \\
0.174\end{array}$ & $\begin{array}{l}0.029 \\
0.052 \\
0.092 \\
0.166\end{array}$ & $\begin{array}{l}0.029 \\
0.051 \\
0.091 \\
0.164\end{array}$ & $\begin{array}{l}0.029 \\
0.053 \\
0.094 \\
0.169\end{array}$ & $\begin{array}{l}0.030 \\
0.054 \\
0.096 \\
0.171\end{array}$ \\
\hline$C_{1}$ & $\begin{array}{l}10^{10} \\
10^{11} \\
10^{12} \\
10^{13}\end{array}$ & $\begin{array}{l}- \\
- \\
- \\
-\end{array}$ & $\begin{array}{l}- \\
- \\
- \\
-\end{array}$ & $\begin{array}{l}- \\
- \\
- \\
-\end{array}$ & $\begin{array}{l}- \\
- \\
-\end{array}$ & $\begin{array}{l}- \\
- \\
- \\
-\end{array}$ & $\begin{array}{l}- \\
- \\
- \\
0.258\end{array}$ & $\begin{array}{l}- \\
- \\
- \\
0.153\end{array}$ & $\begin{array}{l}- \\
- \\
- \\
-\end{array}$ & $\begin{array}{l}- \\
- \\
- \\
-\end{array}$ & $\begin{array}{l}- \\
- \\
- \\
-\end{array}$ \\
\hline$C_{2}$ & $\begin{array}{l}10^{10} \\
10^{11} \\
10^{12} \\
10^{13}\end{array}$ & $\begin{array}{l}0.688 \\
0.688 \\
0.688 \\
0.688\end{array}$ & $\begin{array}{l}0.987 \\
0.987 \\
0.987 \\
0.987\end{array}$ & $\begin{array}{l}1.18 \\
1.18 \\
1.18 \\
1.18\end{array}$ & $\begin{array}{l}1.30 \\
1.30 \\
1.30 \\
1.30\end{array}$ & $\begin{array}{l}1.31 \\
1.31 \\
1.31 \\
1.31\end{array}$ & $\begin{array}{l}0.525 \\
0.525 \\
0.525 \\
0.525\end{array}$ & $\begin{array}{l}0.839 \\
0.839 \\
0.839 \\
0.839\end{array}$ & $\begin{array}{l}1.24 \\
1.24 \\
1.24 \\
1.24\end{array}$ & $\begin{array}{l}1.58 \\
1.58 \\
1.58 \\
1.58\end{array}$ & $\begin{array}{l}1.65 \\
1.65 \\
1.65 \\
1.65\end{array}$ \\
\hline$C_{3}$ & $\begin{array}{l}10^{10} \\
10^{11} \\
10^{12} \\
10^{13}\end{array}$ & $\begin{array}{l}- \\
- \\
-\end{array}$ & $\begin{array}{l}- \\
- \\
- \\
-\end{array}$ & $\begin{array}{l}- \\
- \\
- \\
-\end{array}$ & $\begin{array}{l}- \\
- \\
- \\
-\end{array}$ & $\begin{array}{l}- \\
- \\
-\end{array}$ & $\begin{array}{l}- \\
- \\
-\end{array}$ & $\begin{array}{l}- \\
- \\
- \\
-\end{array}$ & $\begin{array}{l}- \\
- \\
- \\
-\end{array}$ & $\begin{array}{l}- \\
- \\
- \\
-\end{array}$ & $\begin{array}{l}- \\
- \\
- \\
-\end{array}$ \\
\hline$C_{4}$ & $\begin{array}{l}10^{10} \\
10^{11} \\
10^{12} \\
10^{13}\end{array}$ & $\begin{array}{l}0.483 \\
0.483 \\
0.483 \\
0.483\end{array}$ & $\begin{array}{l}0.436 \\
0.436 \\
0.436 \\
0.436\end{array}$ & $\begin{array}{l}0.389 \\
0.389 \\
0.389 \\
0.389\end{array}$ & $\begin{array}{l}0.348 \\
0.348 \\
0.348 \\
0.348\end{array}$ & $\begin{array}{l}0.339 \\
0.339 \\
0.339 \\
0.339\end{array}$ & $\begin{array}{l}0.536 \\
0.536 \\
0.536 \\
0.536\end{array}$ & $\begin{array}{l}0.483 \\
0.483 \\
0.483 \\
0.483\end{array}$ & $\begin{array}{l}0.431 \\
0.431 \\
0.431 \\
0.431\end{array}$ & $\begin{array}{l}0.379 \\
0.379 \\
0.379 \\
0.379\end{array}$ & $\begin{array}{l}0.363 \\
0.363 \\
0.363 \\
0.363\end{array}$ \\
\hline
\end{tabular}


Table 9. Same as Table 1 but for transition $12^{3} \mathrm{~F}-3^{3} \mathrm{D}$ of Helium $(\lambda=8736 \AA)$

\begin{tabular}{|c|c|c|c|c|c|c|c|c|c|c|c|}
\hline & \multirow[b]{2}{*}{$\begin{array}{l}T \\
N_{\mathrm{e}}\end{array}$} & \multirow{2}{*}{ 9a: } & \multirow{2}{*}{$\begin{array}{c}\text { Collisions } \\
2\end{array}$} & \multirow{2}{*}{$\begin{aligned} \text { with } \\
4\end{aligned}$} & \multicolumn{2}{|c|}{ electrons } & \multirow{2}{*}{$\frac{9 b:}{1}$} & Collisions & \multirow[t]{2}{*}{ with } & \multicolumn{2}{|c|}{ protons } \\
\hline & & & & & 8 & 10 & & 2 & & 8 & 10 \\
\hline$W$ & $\begin{array}{l}10^{10} \\
10^{11} \\
10^{12} \\
10^{13}\end{array}$ & $\begin{array}{l}5.06 \mathrm{E}-3 \\
5.06 \mathrm{E}-2 \\
0.506 \\
5.05\end{array}$ & $\begin{array}{l}5.07 \mathrm{E}-3 \\
5.07 \mathrm{E}-2 \\
0.506 \\
5.06\end{array}$ & $\begin{array}{l}5.07 \mathrm{E}-3 \\
5.07 \mathrm{E}-2 \\
0.506 \\
5.06\end{array}$ & $\begin{array}{l}5.06 \mathrm{E}-3 \\
5.06 \mathrm{E}-2 \\
0.506 \\
5.06\end{array}$ & $\begin{array}{l}5.06 \mathrm{E}-3 \\
5.06 \mathrm{E}-2 \\
0.506 \\
5.06\end{array}$ & $\begin{array}{l}5.17 \mathrm{E}-3 \\
5.17 \mathrm{E}-2 \\
0.513 \\
4.72\end{array}$ & $\begin{array}{l}5.16 \mathrm{E}-3 \\
5.15 \mathrm{E}-2 \\
0.514 \\
4.99\end{array}$ & $\begin{array}{l}5.14 \mathrm{E}-3 \\
5.14 \mathrm{E}-2 \\
0.513 \\
5.07\end{array}$ & $\begin{array}{l}5.12 \mathrm{E}-3 \\
5.12 \mathrm{E}-2 \\
0.512 \\
5.09\end{array}$ & $\begin{array}{l}5.11 \mathrm{E}-3 \\
5.11 \mathrm{E}-2 \\
0.511 \\
5.09\end{array}$ \\
\hline$d$ & $\begin{array}{l}10^{10} \\
10^{11} \\
10^{12} \\
10^{13}\end{array}$ & $\begin{array}{l}1.20 \mathrm{E}-5 \\
1.20 \mathrm{E}-4 \\
1.20 \mathrm{E}-3 \\
1.21 \mathrm{E}-2\end{array}$ & $\begin{array}{l}9.85 \mathrm{E}-6 \\
9.85 \mathrm{E}-5 \\
9.85 \mathrm{E}-4 \\
9.85 \mathrm{E}-3\end{array}$ & $\begin{array}{l}7.44 \mathrm{E}-6 \\
7.44 \mathrm{E}-5 \\
7.44 \mathrm{E}-4 \\
7.44 \mathrm{E}-3\end{array}$ & $\begin{array}{l}5.45 \mathrm{E}-6 \\
5.45 \mathrm{E}-5 \\
5.45 \mathrm{E}-4 \\
5.45 \mathrm{E}-3\end{array}$ & $\begin{array}{l}4.96 \mathrm{E}-6 \\
4.96 \mathrm{E}-5 \\
4.96 \mathrm{E}-4 \\
4.96 \mathrm{E}-3\end{array}$ & $\begin{array}{l}1.61 \mathrm{E}-5 \\
1.60 \mathrm{E}-4 \\
1.12 \mathrm{E}-3 \\
4.25 \mathrm{E}-4\end{array}$ & $\begin{array}{l}1.04 \mathrm{E}-5 \\
1.04 \mathrm{E}-4 \\
8.52 \mathrm{E}-4 \\
9.08 \mathrm{E}-4\end{array}$ & $\begin{array}{l}8.33 \mathrm{E}-6 \\
8.33 \mathrm{E}-5 \\
8.26 \mathrm{E}-4 \\
3.05 \mathrm{E}-3\end{array}$ & $\begin{array}{l}7.62 \mathrm{E}-6 \\
7.62 \mathrm{E}-5 \\
7.62 \mathrm{E}-4 \\
5.18 \mathrm{E}-3\end{array}$ & $\begin{array}{l}7.61 \mathrm{E}-6 \\
7.61 \mathrm{E}-5 \\
7.61 \mathrm{E}-4 \\
5.84 \mathrm{E}-3\end{array}$ \\
\hline$A$ & $\begin{array}{l}10^{10} \\
10^{11} \\
10^{12} \\
10^{13}\end{array}$ & & & & & & $\begin{array}{l}0.013 \\
0.0231 \\
0.04 \\
0.073\end{array}$ & $\begin{array}{l}0.013 \\
0.0231 \\
0.04 \\
0.073\end{array}$ & $\begin{array}{l}0.013 \\
0.0231 \\
0.04 \\
0.073\end{array}$ & $\begin{array}{l}0.013 \\
0.0231 \\
0.04 \\
0.073\end{array}$ & $\begin{array}{l}0.013 \\
0.0231 \\
0.04 \\
0.073\end{array}$ \\
\hline$C_{1}$ & $\begin{array}{l}10^{10} \\
10^{11} \\
10^{12} \\
10^{13}\end{array}$ & $\begin{array}{l}- \\
- \\
- \\
0.282\end{array}$ & $\begin{array}{l}- \\
- \\
- \\
0.168\end{array}$ & $\begin{array}{l}- \\
- \\
- \\
-\end{array}$ & $\begin{array}{l}- \\
- \\
-\end{array}$ & $\begin{array}{l}- \\
- \\
- \\
-\end{array}$ & $\begin{array}{l}- \\
0.676 \\
6.76 \\
67.6\end{array}$ & $\begin{array}{l}- \\
0.400 \\
4.00 \\
40.0\end{array}$ & $\begin{array}{l}- \\
0.237 \\
2.37 \\
23.7\end{array}$ & $\begin{array}{l}- \\
0.141 \\
1.41 \\
14.1\end{array}$ & $\begin{array}{l}- \\
0.119 \\
1.19 \\
11.9\end{array}$ \\
\hline$C_{2}$ & $\begin{array}{l}10^{10} \\
10^{11} \\
10^{12} \\
10^{13}\end{array}$ & & & & & & $\begin{array}{l}0.029 \\
0.029 \\
0.029 \\
0.029\end{array}$ & $\begin{array}{l}0.026 \\
0.026 \\
0.026 \\
0.026\end{array}$ & $\begin{array}{l}0.023 \\
0.023 \\
0.023 \\
0.023\end{array}$ & $\begin{array}{l}0.019 \\
0.019 \\
0.019 \\
0.019\end{array}$ & $\begin{array}{l}0.018 \\
0.018 \\
0.018 \\
0.018\end{array}$ \\
\hline$C_{3}$ & $\begin{array}{l}10^{10} \\
10^{11} \\
10^{12} \\
10^{13}\end{array}$ & $\begin{array}{l}112 \\
109 \\
105 \\
100\end{array}$ & $\begin{array}{l}111 \\
109 \\
106 \\
103\end{array}$ & $\begin{array}{l}104 \\
102 \\
101 \\
99\end{array}$ & $\begin{array}{l}92 \\
91 \\
90 \\
88\end{array}$ & $\begin{array}{l}88 \\
75 \\
86 \\
85\end{array}$ & $\begin{array}{l}- \\
- \\
- \\
-\end{array}$ & $\begin{array}{l}- \\
- \\
-\end{array}$ & $\begin{array}{l}- \\
- \\
-\end{array}$ & $\begin{array}{l}- \\
- \\
- \\
-\end{array}$ & $\begin{array}{l}- \\
- \\
- \\
-\end{array}$ \\
\hline$C_{4}$ & $\begin{array}{l}10^{10} \\
10^{11} \\
10^{12} \\
10^{13}\end{array}$ & $\begin{array}{l}0.463 \\
0.463 \\
0.463 \\
0.463\end{array}$ & $\begin{array}{l}0.463 \\
0.463 \\
0.463 \\
0.463\end{array}$ & $\begin{array}{l}0.463 \\
0.463 \\
0.463 \\
0.463\end{array}$ & $\begin{array}{l}0.462 \\
0.462 \\
0.462 \\
0.462\end{array}$ & $\begin{array}{l}0.462 \\
0.462 \\
0.462 \\
0.462\end{array}$ & $\begin{array}{l}0.455 \\
0.455 \\
0.455 \\
0.498\end{array}$ & $\begin{array}{l}0.457 \\
0.457 \\
0.457 \\
0.472\end{array}$ & $\begin{array}{l}0.456 \\
0.456 \\
0.456 \\
0.464\end{array}$ & $\begin{array}{l}0.459 \\
0.459 \\
0.459 \\
0.459\end{array}$ & $\begin{array}{l}0.459 \\
0.459 \\
0.459 \\
0.459\end{array}$ \\
\hline
\end{tabular}

9c: Isolated line approximation validity criterion

\begin{tabular}{|c|c|c|c|c|c|c|}
\hline \multirow{4}{*}{$C_{5}$} & $10^{10}$ & 0.6 & - & - & - & - \\
\hline & $10^{11}$ & 6.37 & - & - & - & - \\
\hline & $10^{12}$ & 63.7 & - & - & - & - \\
\hline & $10^{13}$ & 610.9 & - & - & - & - \\
\hline
\end{tabular}


Table 10. Same as Table 9

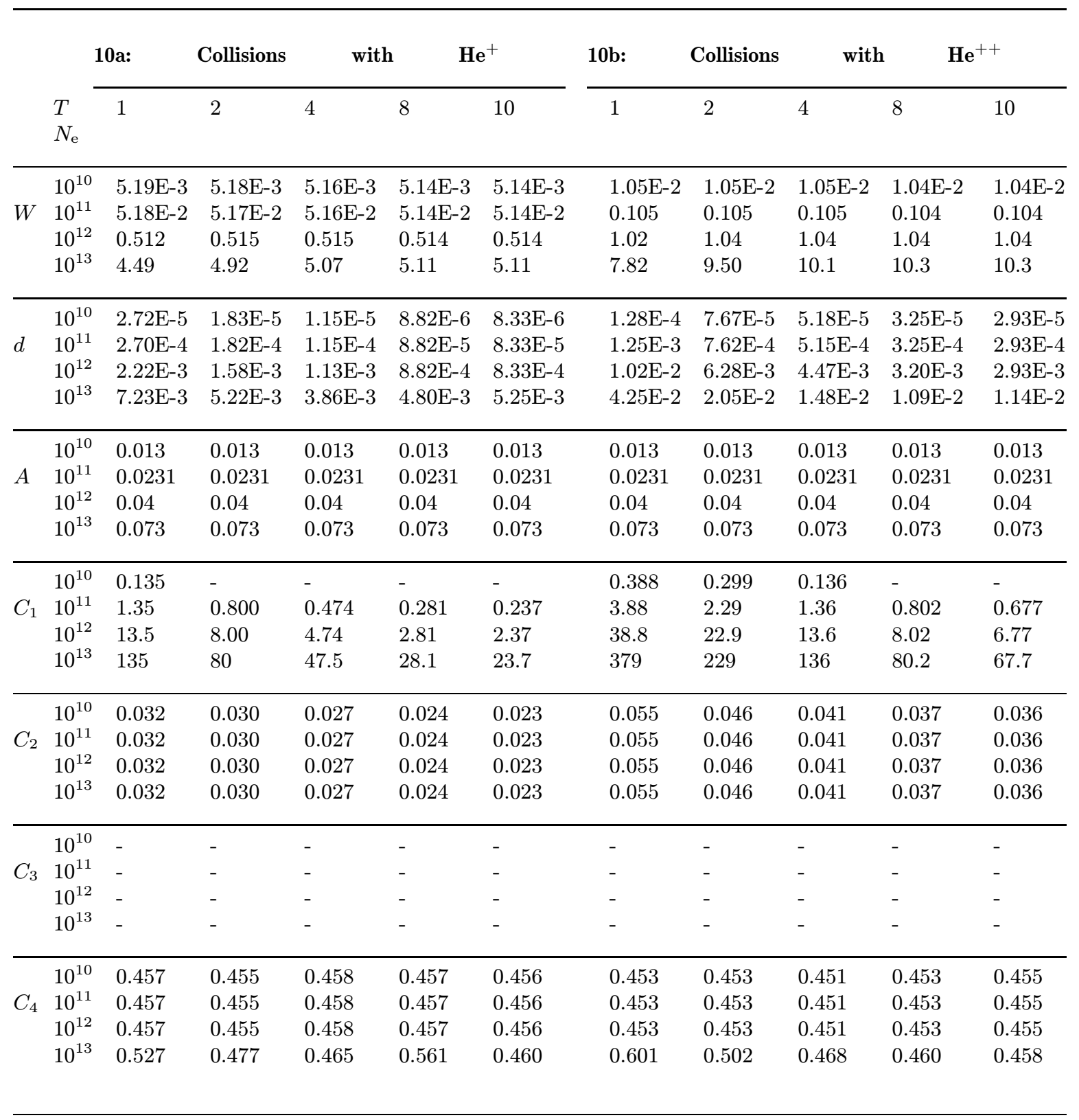


Table 11. Same as Table 1 but for transition $7^{3} \mathrm{~F}-3^{3} \mathrm{D}$ of Helium $(\lambda=10028 \AA)$

\begin{tabular}{|c|c|c|c|c|c|c|c|c|c|c|c|}
\hline & \multirow[b]{2}{*}{$\begin{array}{l}T \\
N_{\mathrm{e}}\end{array}$} & \multirow{2}{*}{ 11a: } & \multirow{2}{*}{$\begin{array}{c}\text { Collisions } \\
2\end{array}$} & \multirow{2}{*}{$\begin{aligned} \text { with } \\
4\end{aligned}$} & \multicolumn{2}{|c|}{ electrons } & \multirow{2}{*}{$\frac{11 b:}{1}$} & \multirow{2}{*}{$\begin{array}{c}\text { Collisions } \\
2\end{array}$} & \multirow[t]{2}{*}{ with } & \multicolumn{2}{|c|}{ protons } \\
\hline & & & & & 8 & 10 & & & & 8 & 10 \\
\hline$W$ & $\begin{array}{l}10^{10} \\
10^{11} \\
10^{12} \\
10^{13}\end{array}$ & $\begin{array}{l}1.63 \mathrm{E}-3 \\
1.60 \mathrm{E}-2 \\
0.143 \\
1.14\end{array}$ & $\begin{array}{l}1.30 \mathrm{E}-3 \\
1.27 \mathrm{E}-2 \\
0.116 \\
0.953\end{array}$ & $\begin{array}{l}1.02 \mathrm{E}-3 \\
1.00 \mathrm{E}-2 \\
0.092 \\
0.779\end{array}$ & $\begin{array}{l}7.94 \mathrm{E}-4 \\
7.92 \mathrm{E}-3 \\
0.072 \\
0.622\end{array}$ & $\begin{array}{l}7.31 \mathrm{E}-4 \\
7.21 \mathrm{E}-3 \\
0.066 \\
0.577\end{array}$ & $\begin{array}{l}3.87 \mathrm{E}-3 \\
3.87 \mathrm{E}-2 \\
0.387 \\
3.86\end{array}$ & $\begin{array}{l}3.80 \mathrm{E}-3 \\
3.80 \mathrm{E}-2 \\
0.380 \\
3.79\end{array}$ & $\begin{array}{l}3.96 \mathrm{E}-3 \\
3.96 \mathrm{E}-2 \\
0.396 \\
3.96\end{array}$ & $\begin{array}{l}3.95 \mathrm{E}-3 \\
3.95 \mathrm{E}-2 \\
0.395 \\
3.94\end{array}$ & $\begin{array}{l}4.01 \mathrm{E}-3 \\
4.01 \mathrm{E}-2 \\
0.401 \\
4.01\end{array}$ \\
\hline$d$ & $\begin{array}{l}10^{10} \\
10^{11} \\
10^{12} \\
10^{13}\end{array}$ & $\begin{array}{l}1.27 \mathrm{E}-5 \\
1.27 \mathrm{E}-4 \\
1.27 \mathrm{E}-3 \\
7.41 \mathrm{E}-3\end{array}$ & $\begin{array}{l}4.66 \mathrm{E}-6 \\
4.66 \mathrm{E}-5 \\
4.66 \mathrm{E}-4 \\
4.66 \mathrm{E}-3\end{array}$ & $\begin{array}{l}1.25 \mathrm{E}-6 \\
1.25 \mathrm{E}-5 \\
1.25 \mathrm{E}-4 \\
1.25 \mathrm{E}-3\end{array}$ & $\begin{array}{l}1.07 \mathrm{E}-6 \\
1.07 \mathrm{E}-5 \\
1.07 \mathrm{E}-4 \\
1.07 \mathrm{E}-3\end{array}$ & $\begin{array}{l}9.91 \mathrm{E}-7 \\
9.91 \mathrm{E}-6 \\
9.91 \mathrm{E}-5 \\
9.91 \mathrm{E}-4\end{array}$ & $\begin{array}{l}2.72 \mathrm{E}-3 \\
2.69 \mathrm{E}-2 \\
0.254 \\
2.75\end{array}$ & $\begin{array}{l}2.76 \mathrm{E}-3 \\
2.74 \mathrm{E}-2 \\
0.266 \\
3.15\end{array}$ & $\begin{array}{l}2.48 \mathrm{E}-3 \\
2.48 \mathrm{E}-2 \\
0.246 \\
3.68\end{array}$ & $\begin{array}{l}1.90 \mathrm{E}-3 \\
1.90 \mathrm{E}-2 \\
0.189 \\
4.34\end{array}$ & $\begin{array}{l}1.58 \mathrm{E}-3 \\
1.58 \mathrm{E}-2 \\
0.157 \\
4.59\end{array}$ \\
\hline$A$ & $\begin{array}{l}10^{10} \\
10^{11} \\
10^{12} \\
10^{13}\end{array}$ & & & & & & $\begin{array}{l}0.375 \\
0.676 \\
1.31 \\
2.75\end{array}$ & $\begin{array}{l}0.445 \\
0.802 \\
1.53 \\
3.15\end{array}$ & $\begin{array}{l}0.533 \\
0.959 \\
1.82 \\
3.68\end{array}$ & $\begin{array}{l}0.643 \\
1.16 \\
2.18 \\
4.34\end{array}$ & $\begin{array}{l}0.684 \\
1.23 \\
2.31 \\
4.59\end{array}$ \\
\hline$C_{1}$ & $\begin{array}{l}10^{10} \\
10^{11} \\
10^{12} \\
10^{13}\end{array}$ & $\begin{array}{l}- \\
- \\
- \\
-\end{array}$ & $\begin{array}{l}- \\
- \\
- \\
-\end{array}$ & $\begin{array}{l}- \\
- \\
- \\
-\end{array}$ & $\begin{array}{l}- \\
- \\
-\end{array}$ & $\begin{array}{l}- \\
- \\
-\end{array}$ & $\begin{array}{l}- \\
0.257 \\
2.57 \\
25.7\end{array}$ & $\begin{array}{l}- \\
0.178 \\
1.78 \\
17.8\end{array}$ & $\begin{array}{l}- \\
- \\
0.591 \\
5.91\end{array}$ & $\begin{array}{l}- \\
- \\
0.435 \\
4.35\end{array}$ & $\begin{array}{l}- \\
- \\
0.354 \\
3.46\end{array}$ \\
\hline$C_{2}$ & $\begin{array}{l}10^{10} \\
10^{11} \\
10^{12} \\
10^{13}\end{array}$ & & & & & & $\begin{array}{l}0.548 \\
0.548 \\
0.548 \\
0.548\end{array}$ & $\begin{array}{l}1.21 \\
1.21 \\
1.21 \\
1.21\end{array}$ & $\begin{array}{l}2.5 \\
2.5 \\
2.5 \\
2.5\end{array}$ & $\begin{array}{l}6.64 \\
6.64 \\
6.64 \\
6.64\end{array}$ & $\begin{array}{l}8.00 \\
8.0 \\
8.0 \\
8.0\end{array}$ \\
\hline$C_{3}$ & $\begin{array}{l}10^{10} \\
10^{11} \\
10^{12} \\
10^{13}\end{array}$ & $\begin{array}{l}- \\
- \\
- \\
-\end{array}$ & $\begin{array}{l}- \\
- \\
- \\
-\end{array}$ & $\begin{array}{l}- \\
- \\
- \\
-\end{array}$ & $\begin{array}{l}- \\
- \\
- \\
-\end{array}$ & $\begin{array}{l}- \\
- \\
-\end{array}$ & $\begin{array}{l}- \\
- \\
- \\
-\end{array}$ & $\begin{array}{l}- \\
- \\
- \\
-\end{array}$ & $\begin{array}{l}- \\
- \\
- \\
-\end{array}$ & $\begin{array}{l}- \\
- \\
- \\
-\end{array}$ & $\begin{array}{l}- \\
- \\
- \\
-\end{array}$ \\
\hline$C_{4}$ & $\begin{array}{l}10^{10} \\
10^{11} \\
10^{12} \\
10^{13}\end{array}$ & $\begin{array}{l}0.090 \\
0.092 \\
0.10 \\
0.128\end{array}$ & $\begin{array}{l}0.081 \\
0.082 \\
0.09 \\
0.110\end{array}$ & $\begin{array}{l}0.073 \\
0.075 \\
0.08 \\
0.097\end{array}$ & $\begin{array}{l}0.067 \\
0.068 \\
0.07 \\
0.086\end{array}$ & $\begin{array}{l}0.066 \\
0.067 \\
0.07 \\
0.084\end{array}$ & $\begin{array}{l}0.422 \\
0.422 \\
0.422 \\
0.422\end{array}$ & $\begin{array}{l}0.476 \\
0.476 \\
0.476 \\
0.476\end{array}$ & $\begin{array}{l}0.309 \\
0.309 \\
0.309 \\
0.309\end{array}$ & $\begin{array}{l}0.358 \\
0.358 \\
0.358 \\
0.358\end{array}$ & $\begin{array}{l}0.337 \\
0.337 \\
0.337 \\
0.337\end{array}$ \\
\hline
\end{tabular}

11c: Isolated line approximation validity criterion

\begin{tabular}{|c|c|c|c|c|c|c|}
\hline & $10^{10}$ & 0.02 & - & - & - & - \\
\hline$C_{5}$ & $10^{11}$ & 0.28 & 0.25 & 0.25 & 0.23 & 0.23 \\
\hline & $10^{12}$ & 2.7 & 2.5 & 2.4 & 2.3 & 2.3 \\
\hline & $10^{13}$ & 25.2 & 23.7 & 23.9 & 23.05 & 22.6 \\
\hline
\end{tabular}


Table 12. Same as Table 11

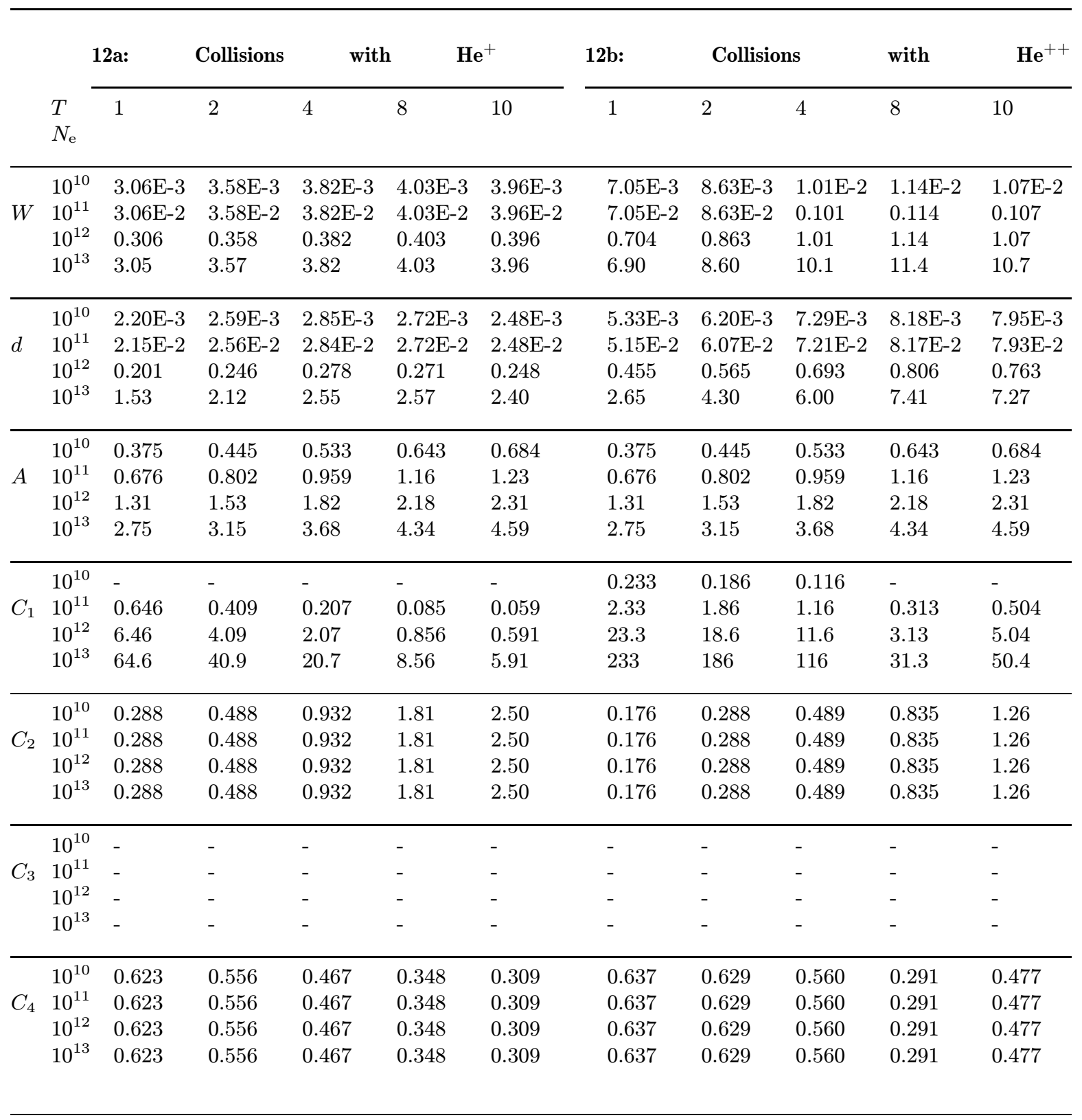

